\title{
12. CALCAREOUS NANNOPLANKTON BIOSTRATIGRAPHY OF THE SOUTHERN CORAL SEA, TASMAN SEA, AND SOUTHWESTERN PACIFIC OCEAN, DEEP SEA DRILLING PROJECT LEG 90: NEOGENE AND QUATERNARY1
}

\author{
William H. Lohman, Marathon Oil Company ${ }^{2}$
}

\begin{abstract}
Leg 90 of the Deep Sea Drilling Project drilled 18 holes at eight sites (Sites 587-594) on several shallow-water platforms in the southern Coral Sea, Tasman Sea, and southwestern Pacific Ocean. The results from an additional hole (Hole 586B) drilled at Site 586 during Leg 89 are included in this report. Together, these sites form a latitudinal traverse which extends from the equator (Site 586) to $45^{\circ} \mathrm{S}$ (Site 594) and includes all the major water masses from tropical to subantarctic. Samples recovered at these sites range in age from middle Eocene to late Quaternary. The calcareous nannoplankton biostratigraphy for Leg 90 has divided into two parts: part 1, the Neogene and Quaternary of Sites 586-594. (this chapter); and part 2, the Paleogene of Sites 588, 592, and 593 (Martini, this volume). A slightly modified version of the Martini (1971) standard Tertiary and Quaternary zonation scheme was used to make age determinations on over 700 samples. All of the relevant Neogene and Quaternary zone-defining nannoplankton are present at Sites 586-591 $\left(0^{\circ}-30^{\circ} \mathrm{S}\right)$ but become increasingly rare or are absent at Sites $592-594\left(35^{\circ}-45^{\circ} \mathrm{S}\right)$. Species diversity increases southward from the equator (Site 586) and reaches a peak at $20^{\circ} \mathrm{S}$ (Site 587). A decrease at $25^{\circ} \mathrm{S}$ (Site 588) and $30^{\circ} \mathrm{S}$ (Sites 589-591) is followed by an increase in species diversity at $35^{\circ} \mathrm{S}$ (Site 592). South of $35^{\circ} \mathrm{S}$, species diversity again decreases and reaches a low at $45^{\circ} \mathrm{S}$ (Site 594). Species diversity for all sites as a group generally increases through the early, middle, and late Miocene, reaches a peak in the early Pliocene, then gradually decreases through the late Pliocene and Quaternary.
\end{abstract}

\section{INTRODUCTION}

The southwest Pacific, unlike most other oceanic regions, contains numerous submarine topographic features. These bathymetric highs, or platforms, extend latitudinally over vast distances and are overlain by relatively uncomplicated middle to upper Cenozoic calcareous ooze and chalk, free of terrigenous content. One of the major platforms in the region, the Lord Howe Rise, is approximately $300 \mathrm{~km}$ wide and extends some $2000 \mathrm{~km}$ northward from central western New Zealand to about $20^{\circ} \mathrm{S}$. The crest lies at water depths ranging from 750 to $1200 \mathrm{~m}$.

Leg 90 of the Deep Sea Drilling Project (Noumea, New Caledonia, to Wellington, New Zealand) occupied eight sites (Fig. 1) in the southern Coral Sea, Tasman Sea, and southwestern Pacific east of New Zealand's South Island during December, 1982, and January, 1983. The sites are located on shallow-water platforms extending south from the southern slope of Lansdowne Bank (Site 587), northern to southern Lord Howe Rise (Sites 588-592), Challenger Plateau (Site 593), and the southern margin of the Chatham Rise (Site 594). Two methods of drilling, the hydraulic piston corer (HPC) and the extended core barrel (XCB), were used during Leg 90 to drill 18 holes in water depths ranging from $1068 \mathrm{~m}$ (from sea level) at Site 593 to $2131 \mathrm{~m}$ at Site 591. The $\mathrm{XCB}$, first successfully used during Leg 90 , is a core

\footnotetext{
${ }^{1}$ Kennett, J. P., von der Borch, C. C., et al., Init. Repts. DSDP, 90: Washington (U.S. Govt, Printing Office)

2 Address: Marathon Oil Company, Denver Research Center, P.O. Box 269, Littleton, CO 80160
}

barrel that extends several centimeters ahead of the main rotary drill pipe bit and is used to core sediments after they have become too consolidated for coring with the HPC. The use of the HPC and XCB methods permitted greater core recovery with much less disturbance than with standard rotary coring and, as a result, a record 495 high-quality cores representing over $3700 \mathrm{~m}$ of sediment were recovered. An additional equatorial site (Site 586), located on the Ontong-Java Plateau at a water depth of $2207 \mathrm{~m}$ (from sea level), was drilled during Leg 89 , and the 25 cores recovered from Hole 586B were set aside for study by Leg 90 scientists. The results from Hole 586B are included in the Leg 90 report. Samples recovered at these sites range in age from middle Eocene to late Quaternary. Only three sites (Sites 588, 592, and 593) penetrated pre-Miocene sediment.

The calcareous nannoplankton biostratigraphy for Leg 90 has been divided into two reports: part 1, the Neogene and Quaternary of Sites 586-594 (this chapter); and part 2, the Paleogene of Sites 588, 592, and 593 (Marti$\mathrm{ni}$, this volume). Over 700 samples from the nine sites were examined for calcareous nannoplankton. All of the relevant Neogene and Quaternary zone-defining species used during Leg 90 are present at Sites 586-591 $\left(0^{\circ}-\right.$ $30^{\circ} \mathrm{S}$ ) but become increasingly rare or are absent at Sites $592-594\left(35^{\circ}-45^{\circ} \mathrm{S}\right)$. The nannoplankton age determinations of Leg 90 samples made during this study agree very closely with those determined aboard the ship. Latitudinal effects on calcareous nannoplankton diversity are reflected in the changes in numbers of indigenous species observed from site to site along the traverse. Indigenous species range from a high of 48 in the early Pliocene at Site $587\left(20^{\circ} \mathrm{S}\right)$ to a low of 13 in the late Pliocene at Site $594\left(45^{\circ} \mathrm{S}\right)$. 


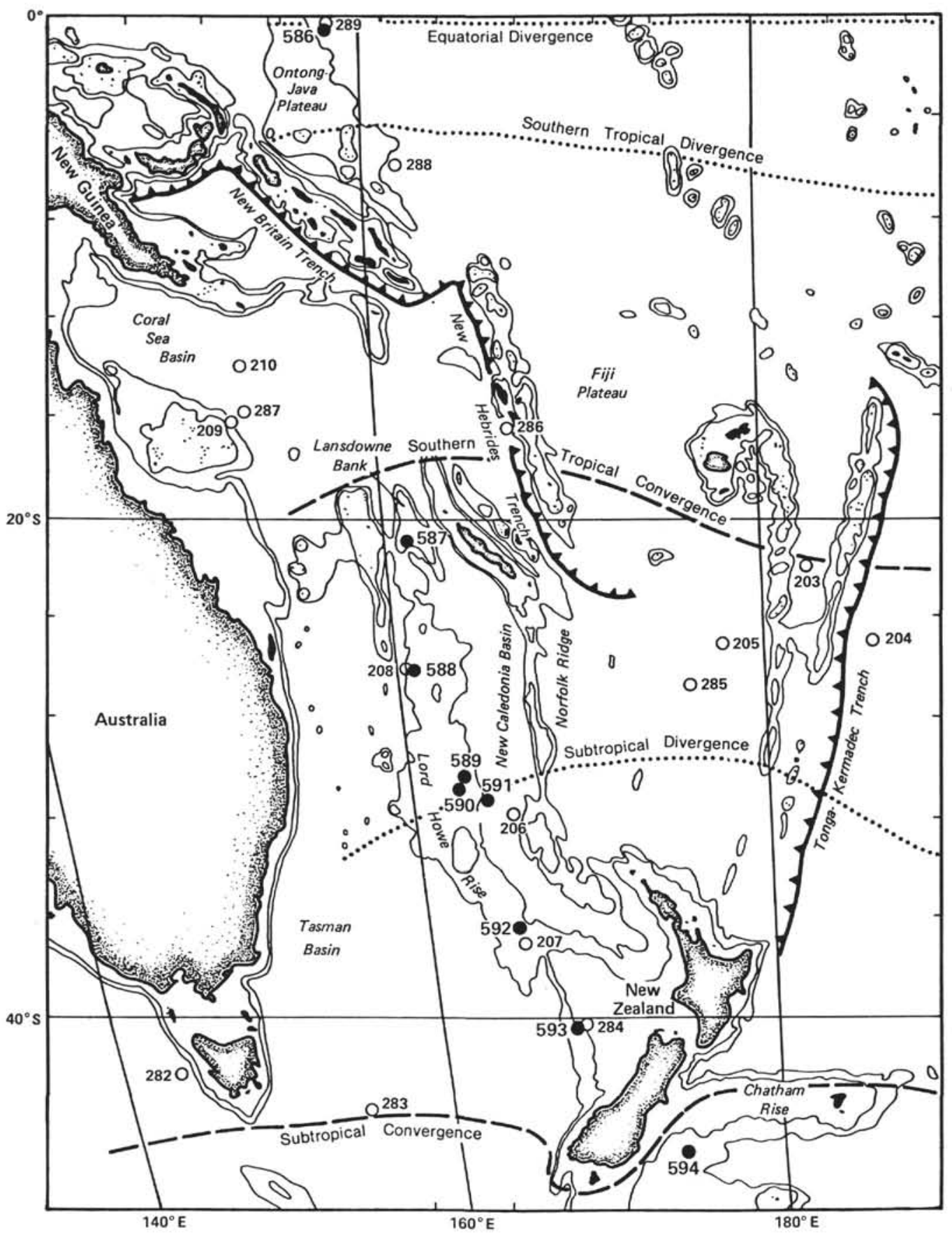

Figure 1. DSDP drill sites from Leg 90 and other DSDP legs in the southwest Pacific.

\section{NANNOPLANKTON ZONATION}

A slightly modified version of the Martini (1971) standard Tertiary and Quaternary zonation scheme (Fig. 2) was used to make the initial calcareous nannoplankton age determinations during Leg 90 . For consistency, the zonation scheme used aboard ship is also used in this chapter.

The zonal modifications include the subdivision of Zones NN19 and NN11 and the use of an alternate species to define the upper boundary of Zone NN4. The last occurrence of Calcidiscus macintyrei occurs within the early Quaternary Emiliania ovata (= Pseudoemilia- nia lacunosa) Zone (NN19) and is used to subdivide Zone NN19 into Subzones NN19a and NN19b. Subzone $\mathrm{NN19a}$ is equivalent to the Calcidiscus macintyrei (= Cyclococcolithina macintyrei) Zone of Gartner (1977) and Subzone WPN-30a of Ellis (1982). Subzone NN19b is equivalent to Subzones WPN-31a and WPN-30b of Ellis (1982). The late Miocene Discoaster quinqueramus Zone (NN11) is subdivided into Subzones NN11a and NN11b by the first occurrence of Amaurolithus primus within Zone NN11. Subzone NN11b is equivalent to the Amaurolithus primus (= Ceratolithus primus) Subzone of Bukry (1973b), Subzone CN9b of Okada and Bukry (1980), and Subzone WPN-26b of Ellis (1982). Subzone 


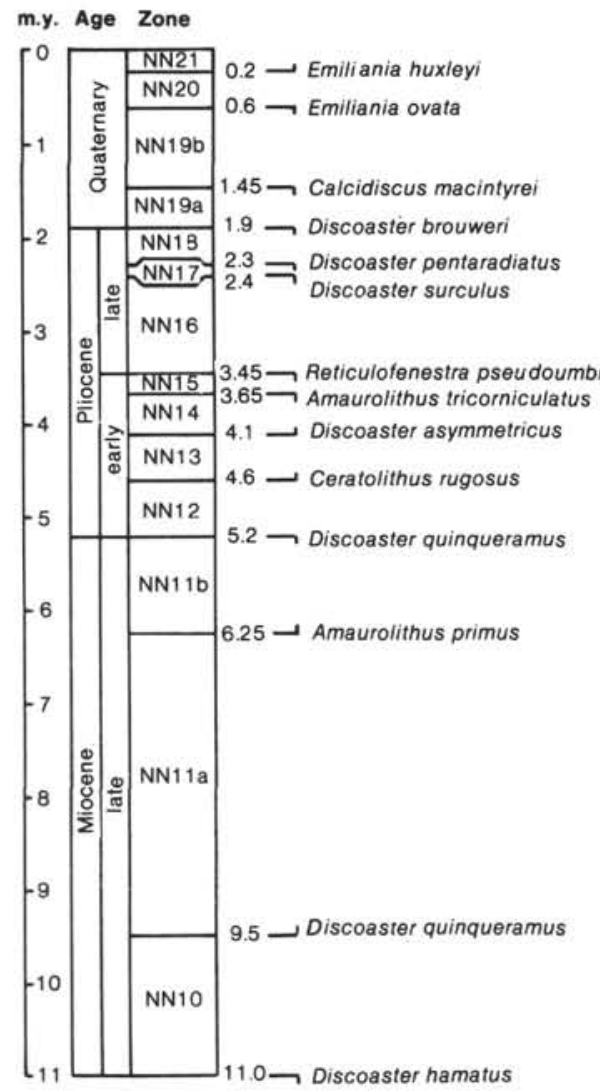

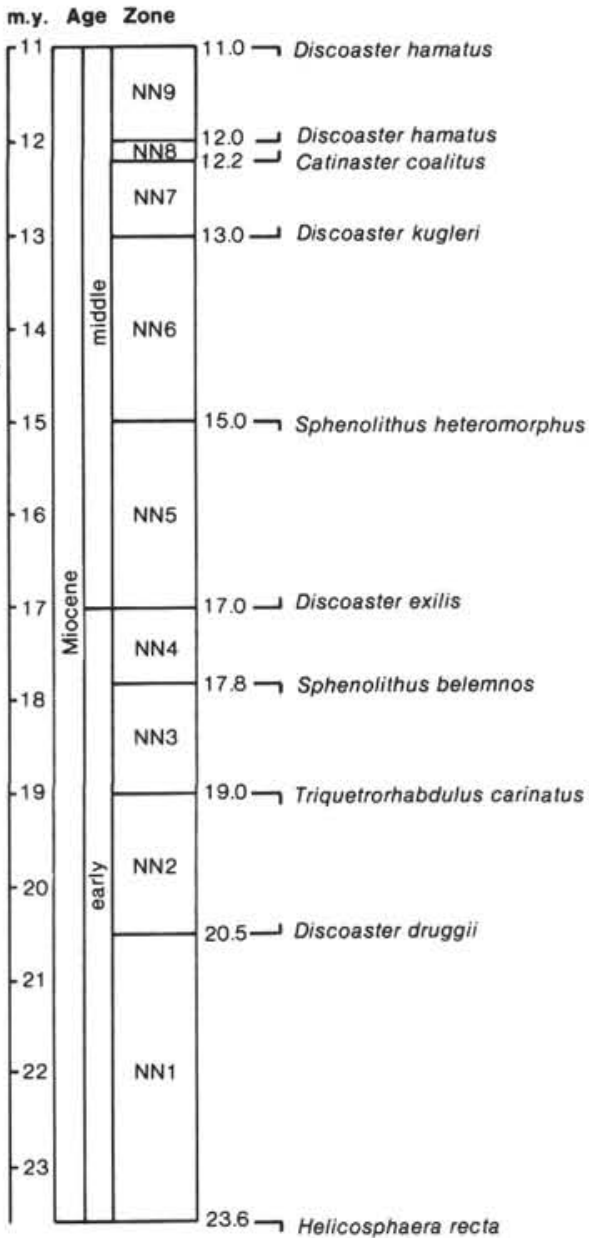

Figure 2. Calcareous nannoplankton zonation scheme used for the Quaternary and Neogene of Leg 90; modified after Martini (1971). Ages for zonal boundaries are standardized for Leg 90 (Martini, this volume).

NN11a is equivalent to Subzone WPN-26a of Ellis (1982). Because Helicosphaera ampliaperta is apparently absent in the western Pacific, the upper boundary of the early Miocene Helicosphaera ampliaperta Zone (NN4), normally placed at the last occurrence of the nominal species, is instead marked by the first occurrence of Discoaster exilis, which closely approximates this level (Marti$\mathrm{ni}$ and Worsley, 1971).

\section{NANNOPLANKTON ABUNDANCE AND DIVERSITY}

The locations of Sites 587-594 (Leg 90), together with Site 586 (Leg 89), form a latitudinal traverse which extends from the equator to $45^{\circ} \mathrm{S}$ and includes all the major surface-water masses between the tropics and the sub-Antarctic. The effects of higher latitudes and coolerwaters are reflected in the changes in abundance and diversity of calcareous nannoplankton observed at sites along this traverse.

The average abundance of each of the Neogene and Quaternary zonal and subzonal indicators at various southern latitudes is shown in Figure 3. The vertical axis indicates abundance in terms of $\mathrm{A}=$ abundant, $\mathrm{C}=$ common, $\mathrm{R}=$ rare, and $\mathrm{B}=$ barren (for quantitative estimates of $A, C, R$, see below). The abundance values for each taxon were obtained by assigning a value of $\mathrm{A}$ $=3, \mathrm{C}=2, \mathrm{R}=1$, or $\mathrm{B}=\mathrm{O}$ for each occurrence on the nannoplankton range charts and calculating the numerical average for each taxon at each site. The horizontal axis includes each of the nine sites plotted to the nearest increment of $5^{\circ} \mathrm{S}$ latitude. Abundance values for taxa at $30^{\circ} \mathrm{S}$ are the average of those obtained at Sites 589,590 , and 591. For some taxa there are no data in some sections, because drilling operations ended at those sites before the sections were penetrated. In general, the abundance of zone-defining discoasters declines steadily at higher latitudes; they are extremely rare at $45^{\circ} \mathrm{S}$. However, three discoasters (Discoaster pentaradiatus, $D$. asymmetricus, and $D$. quinqueramus) reach an abundance peak at $25^{\circ} \mathrm{S}$ before declining sharply. The abundances of other zone-defining species, including Amaurolithus primus, A. tricorniculatus, Calcidiscus macintyrei, $\mathrm{He}$ licosphaera recta, Reticulofenestra pseudoumbilica, and Triquetrorhabdulus carinatus appear to change very little with latitude. The abundance of one species, Emiliania huxleyi, increases with latitude, reaching a peak at $40^{\circ} \mathrm{S}$.

Changes in nannoplankton diversity for indigenous species at various southern latitudes are shown in Figure 4 . The highest diversity of nannoplankton (33 species) in Quaternary sediments occurs at $20^{\circ} \mathrm{S}$ (Site 587). 

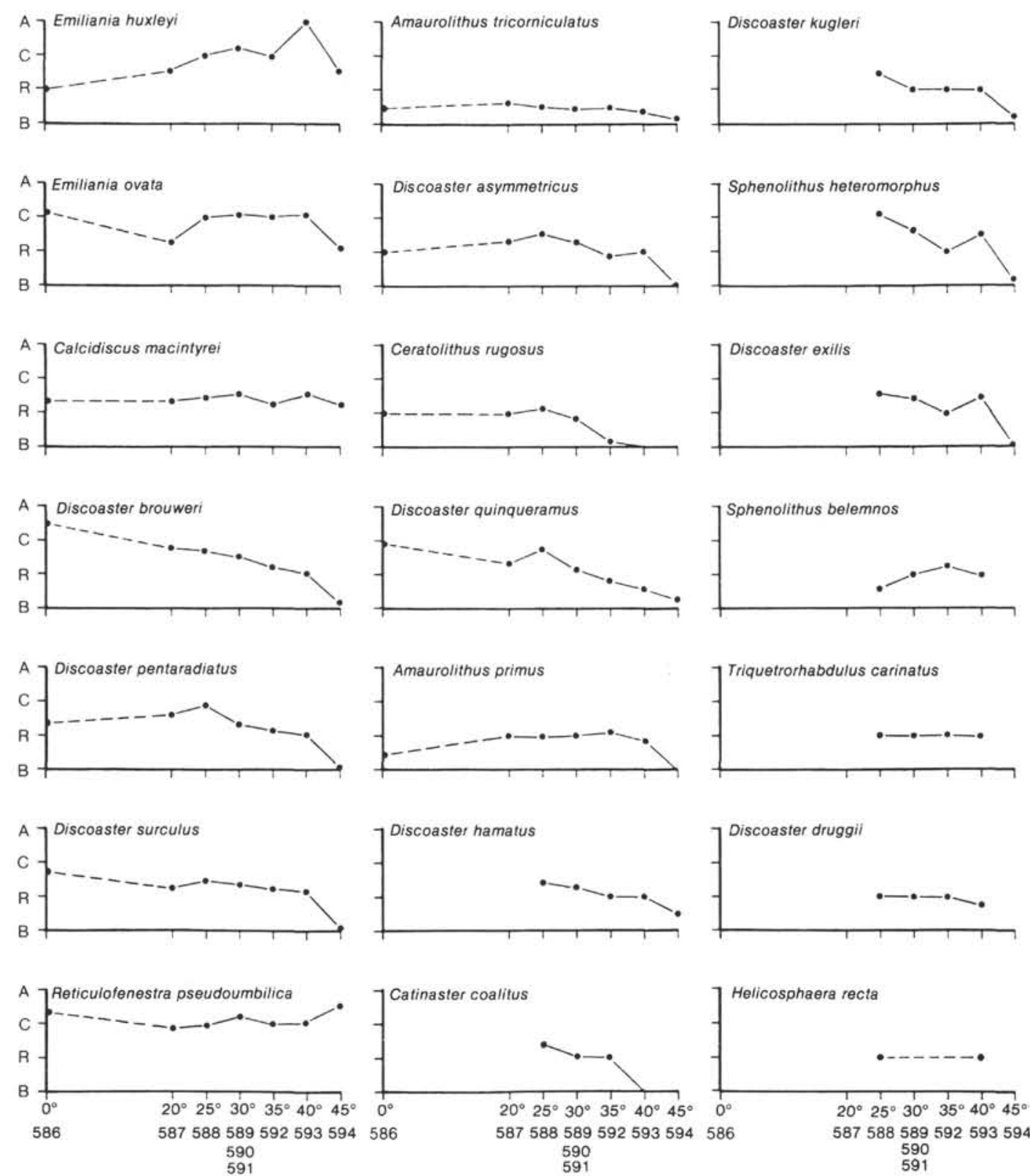

Figure 3. Relative abundances ( ) of Quaternary and Neogene zonal and subzonal indicators encountered at various southern latitudes. Dashed lines indicate latitudes for which there are no data. Vertical axis: $\mathrm{A}=$ abundant, $\mathrm{C}=$ common, $\mathrm{R}=$ rare, and $\mathrm{B}=$ barren (fully defined in text). Horizontal axis: Approximate southern latitude of sites.

South of Site 587 , there is a steady decline in diversity to a low of 20 species at $45^{\circ} \mathrm{S}$ (Site 594). In the late and early Pliocene and in the late Miocene, diversity peaks also occur at $20^{\circ} \mathrm{S}$ (Site 587), with a maximum of 48 species in the early Pliocene, South of Site 587, a decline in diversity at $25^{\circ} \mathrm{S}$ and $30^{\circ} \mathrm{S}$ (Sites 588-591) is followed by peaks of lesser diversity at $35^{\circ} \mathrm{S}$ (Site 592). The lowest diversity (13 species) occurs in the late Pliocene at $45^{\circ} \mathrm{S}$ (Site 594). The middle Miocene at Sites 588 and 590-594 and the early Miocene at Sites 588, 590, and 592-593 are marked by lower diversity.

The data presented in Figures 3 and 4 point out certain latitudinal abundance and diversity trends observed during Leg 90 and show the limitations of calcareous nannoplankton for zoning sediments south of $40^{\circ} \mathrm{S}$.

\section{SYSTEMATIC PALEONTOLOGY}

Forty genera and 122 species of calcareous nannoplankton were recognized during the study of core samples from Sites 586-594 (Fig. 5 ). The genera are listed alphabetically for convenient reference and a brief synonomy of indigenous and reworked species is included.

\section{Genus AMAUROLITHUS Gartner and Bukry, 1975}

\section{Amaurolithus amplificus (Bukry and Percival)}

Ceratolithus amplificus Bukry and Percival, 1971, p. 125, pl. 1, figs. 9-11.

Amaurolithus amplificus (Bukry and Percival). Gartner and Bukry, 1975 , pp. $455-456$, figs. $6 \mathrm{~g}-1$.

\section{Amaurolithus bizzarus (Bukry)}

Ceratolithus bizzarus Bukry, 1973a, p. 676, pl. 1, figs. 6-10. Amaurolithus bizzarus (Bukry). Gartner and Bukry, 1975, p. 456, figs. $8 \mathrm{a}-\mathrm{b}$. 


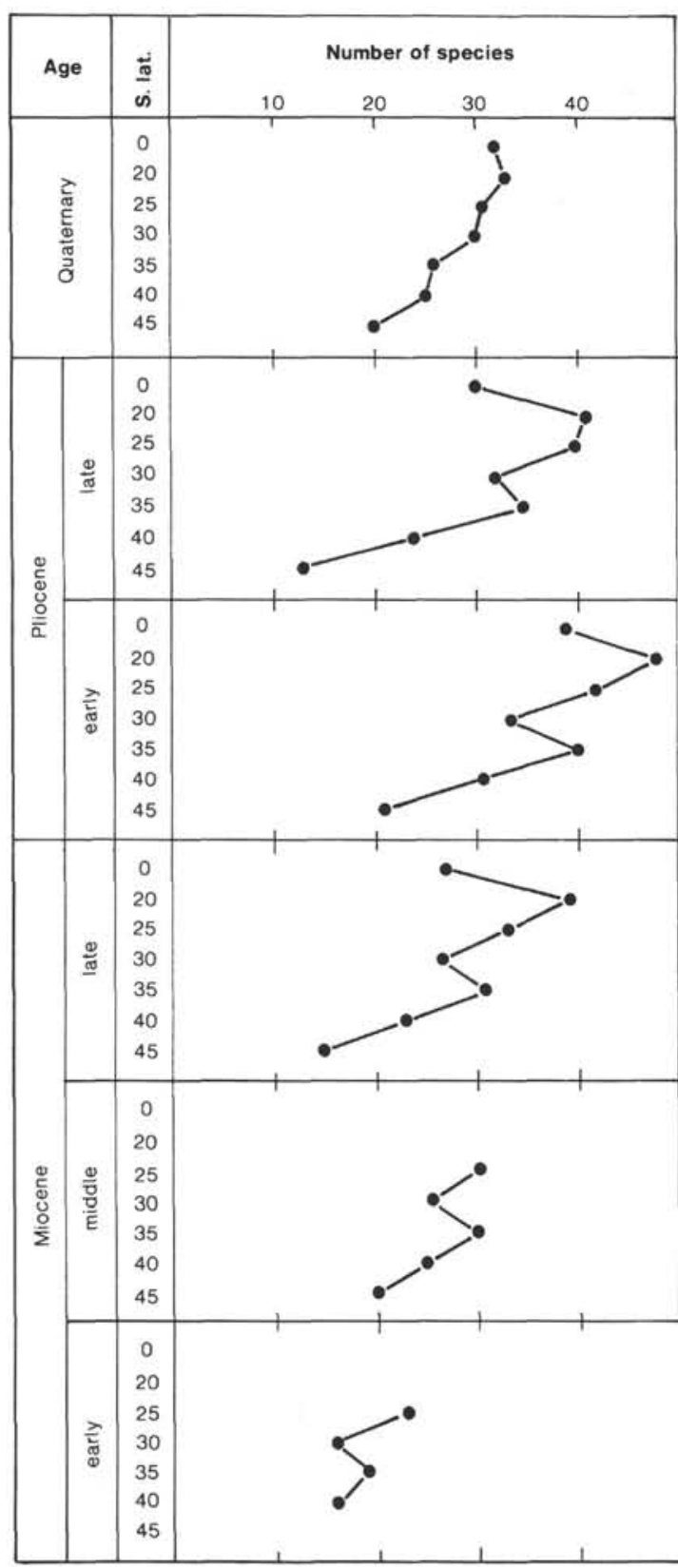

Figure 4. Total number of indigenous calcareous nannoplankton species observed in the Quaternary and Neogene at various southern latitudes.

\section{Amaurolithus delicatus Gartner and Bukry}

Amaurolithus delicatus Gartner and Bukry, 1975, p. 456, figs. 7a-f.

\section{Amaurolithus primus (Bukry and Percival)}

Ceratolithus primus Bukry and Percival, 1971, p. 126, pl. 1, figs. 12-14.

Amaurolithus primus (Bukry and Percival). Gartner and Bukry, 1975, p. 457 , figs. $7 \mathrm{~g}-1$.

\section{Amaurolithus tricorniculatus (Gartner)}

Ceratolithus tricorniculatus Gartner, 1967, p. 5, pl. 10, figs. 4-6. Amaurolithus tricorniculatus (Gartner). Gartner and Bukry, 1975, pp. $457-458$, figs. $8 \mathrm{c}-\mathrm{h}$.
Genus ANGULOLITHINA Bukry, 1973

Angulolithina arca Bukry

Angulolithina arca Bukry, 1973a, p. 675, pl. 1, figs. 1-5.

Genus BRAARUDOSPHAERA Deflandre, 1947

Braarudosphaera bigelowii (Gran and Braarud)

Pontosphaera bigelowi Gran and Braarud, 1935, p. 389, fig. 67.

Braarudosphaera bigelowi (Gran and Braarud). Deflandre, 1947, p. 439 , figs. $1-5$.

Genus CALCIDISCUS Kamptner, 1950

Calcidiscus leptoporus (Murray and Blackman)

Coccosphaera leptopora Murray and Blackman, 1898, p. 430, pl. 15, figs. 1-7.

Calcidiscus leptoporus (Murray and Blackman). Loeblich and Tappan, 1978, p. 1391.

\section{Calcidiscus macintyrei (Bukry and Bramlette)}

Cyclococcolithus macintyrei Bukry and Bramlette, 1969, p. 132, pl. 1, figs. 1-3.

Calcidiscus macintyrei (Bukry and Bramlette). Loeblich and Tappan, 1978, p. 1392.

\section{Genus CATINASTER Martini and Bramlette, 1963}

Catinaster calyculus Martini and Bramlette

Catinaster calyculus Martini and Bramlette, 1963, p. 850, pl. 103, figs. 1-6.

\section{Catinaster coalitus Martini and Bramlette}

Catinaster coalitus Martini and Bramlette, 1963, p. 851, pl. 103, figs. 7-10.

\section{Genus CERATOLITHUS Kamptner, 1950}

Ceratolithus acutus Gartner and Bukry

Ceratolithus acutus Gartner and Bukry, 1974, pl. 115, pl. 1, figs. 1-4.

\section{Ceratolithus armatus Müller}

Ceratolithus armatus Müller, 1974, pl. 591, pl. 11, figs. 4-6; pl. 19, figs. 3-4.

\section{Ceratolithus cristatus Kamptner}

Ceratolithus cristatus Kamptner, 1954, p. 43, figs. 44-45.

\section{Ceratolithus rugosus Bukry and Bramlette}

Ceratolithus rugosus Bukry and Bramlette, 1968, p. 152, pl. 1, figs. 5-9.

\section{Ceratolithus telesmus Norris}

Ceratolithus telesmus Norris, 1965, p. 21, pl. 11, figs. 5-7; pl. 13, figs. 1-3.

\section{Genus CHIASMOLITHUS Hay, Mohler, and Wade, 1966}

Chiasmolithus altus Bukry and Percival

Chiasmolithus altus Bukry and Percival, 1971, p. 126, pl. 2, figs. 1-2.

Chiasmolithus bidens (Bramlette and Sullivan)

Coccolithus bidens Bramlette and Sullivan, 1961, p. 139, pl. 1, fig. 1. Chiasmolithus bidens (Bramlette and Sullivan). Hay and Mohler, 1967, p. 1526 , pl. 196, figs. 23-25.

\section{Chiasmolithus californicus (Sullivan)}

Coccolithus californicus Sullivan, 1964, p. 180, pl. 2, figs. 3a-b, 4a-b. Chiasmolithus californicus (Sullivan). Hay and Mohler, 1967, p. 1527, pl. 196, figs. 18-20; pl. 198, fig. 5 . 


\begin{tabular}{|c|c|c|c|c|c|c|c|c|c|c|c|c|c|c|c|c|c|c|c|c|c|c|c|c|c|c|c|c|c|c|}
\hline \multirow[b]{2}{*}{ Genus } & \multirow[b]{2}{*}{ Species } & \multicolumn{8}{|c|}{ Site } & \multirow[b]{2}{*}{ Genus } & \multirow[b]{2}{*}{ Species } & \multicolumn{8}{|c|}{ Site } & \multirow[b]{2}{*}{ Genus } & \multirow[b]{2}{*}{ Species } & \multicolumn{9}{|c|}{ Site } \\
\hline & & : & & 巴 & & & 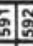 & & 包费 & & & 边 & & : & : & 8 & & 召 & & & & 忑 & T: & & & & & & & $\overline{8}$ \\
\hline \multirow[t]{5}{*}{ Amaurolithus } & amplificus & - & - & - & & & 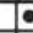 & 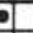 & & \multirow[t]{19}{*}{ Discoaster } & deflandrei & & & - & 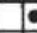 & 10 & 10 & & $\bullet$ & Lophodolithus & nascens & & & & & & & & & $x$ \\
\hline & bizzarus & & - & & & & & & & & druggii & & & - & - & a & e & - & & Oolithotus & fragilis & - & - & - & - & - & - & • & - & \\
\hline & delicatus & & - & - & & -10 & e. & - & & & exilis & & $x$ & - & 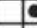 & 9 & 10 & - & e) & Orthorhabdus & serratus & & & e & & & - & & & \\
\hline & primus & $\bullet$ & $\bullet$ & - & & $\bullet$ & e- & - & & & hamatus & & & - & & - & - & $\bullet$ & e & Pontosphaera & discopora & $\bullet$ & $\bullet$ & $\bullet$ & - & - & $\bullet$ & $\bullet$ & - & - \\
\hline & tricorniculatus & $\bullet$ & - & - & & $\bullet$ & ele & - & $\bullet$ & & \begin{tabular}{|l} 
intercalaris \\
\end{tabular} & & & & & & & & $x$ & Reticulofenestra & hillae & & & & & & & & & $x$ \\
\hline Anguiolithina & arca & & -1 & & & & & & & & kugleni & & & - & & e & & - & 리 & & pseudoumbilica & e & $\bullet$ & - & $\mathbf{x}$ & e & $\bullet$ & $\bullet$ & - & 라 \\
\hline Braarudosphaera & bigeiowii & & - & & & & & & & & lodoensis & & & & & & & & $x$ & & umbilica & & & $x$ & & & & $x$ & & $\bar{x}$ \\
\hline \multirow[t]{2}{*}{ Calcidiscus } & leptoporus & e & e & - & - & -1 & ๑e & 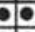 & $\theta$ & & Ioeblichii & & $x$ & & & & & & & Rhabdosphaera & clavigera & - & $\bullet$ & - & 바 & - & $\bullet$ & - & $\bullet$ & \\
\hline & macintyrei & 인 & e & 리 & -1 & $\bullet$ & e. & . & 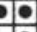 & & moorei & $x$ & & 다 & & 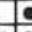 & & e & & & procera & e & $\bullet$ & e. & ㄹ. & - & - & - & - & 은 \\
\hline Catinaster & calyculus & & & $\bullet$ & & - & & & 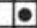 & & neohamatus & & - & - & 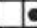 & 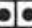 & e & - & $\bullet$ & Scapholithus & fossilis & • & - & - & $\bullet$ & $\bullet$ & - & - & - & \\
\hline & coalitus & & & - & & - & -10 & • & & & pentaradiatus & - & - & - & $x \mid$ & 0 & 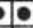 & - & • & Scyphosphaera & amphora & - & & & & & - & & & \\
\hline Ceratolithus & acufus & e & & - & & & & - & - & & perclarus & & & & & & e & & & & apsteinii & - & $\bullet$ & & & $\bullet$ & & & & \\
\hline & armatus & & - & & & & & & & & quinqueramus & - & - & - & e & 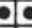 & - & - & - & & campanula & & $\bullet$ & & & & & & & \\
\hline & cristatus & e. & -1 & - & - & -1 & ele & e & & & stellulus & & & & & 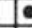 & & & • & & conica & & $\bullet$ & & & - & & & & \\
\hline & rugosus & - & -1 & - & - & -1) & e & e & & & surculus & - & - & - & $x=$ & 0 & - & e & • & & cylindrica & & $\bullet$ & & & & - & & & \\
\hline & telesmus & - & - & - & - & - & - & e & & & tamalis & - & - & - & $\times$ & -e & - & - & & & globulata & e & - & - & & - & - & - & & \\
\hline Chiasmolithus & altus & & & & & & & $x$ & $x$ & & \begin{tabular}{|l} 
tridenus \\
\end{tabular} & - & $\bullet$ & - & & - e & $\bullet$ & - & & & intermedia & e & - & - & & $\bullet$ & - & $\bullet$ & $\bullet$ & \\
\hline & bidens & & & & & & & & $x$ & & \begin{tabular}{|l} 
triradiatus \\
\end{tabular} & - & - & - & - 1 & ec & - & - & - & & \begin{tabular}{|l} 
magna \\
\end{tabular} & & $\bullet$ & - & - & - & - & - & & \\
\hline & californicus & & & & & & & & $x$ & & variabilis & er & $\bullet$ & - & $\times=$ & - & e & e & • & & pulcherrima & e & • & e & - & - & - & - & - & \\
\hline & grandis & & & & & & & & $x$ & Discolithina & japonica & $\bullet$ & $\bullet$ & - & •? & - $\bullet$ & $\bullet$ & $\bullet$ & e & & recurvata & e & • & - & $\bullet$ & - & - & - & $\bullet$ & - \\
\hline & oamaruensis & & & & & & & & $x$ & & multipora & - & & & & & & $\bullet$ & e) & Sphenolithus & abies & e & - & - & & - & - & - & & 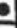 \\
\hline Coccolithus & carteri & e) & 라 & $\bullet$ & - & - & - & - e & - & Discosphaera & tubifera & & - & - & -10 & - & & & & & belemnos & & & - & & - & & & - & \\
\hline & miopelagicus & & & e & & - & 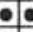 & 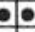 & 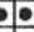 & Emiliania & annula & - & $\bullet$ & - & $\bullet$ & $\bullet$ & 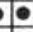 & - & & & \begin{tabular}{|c|c|} 
capricornutus \\
\end{tabular} & & & $\bullet$ & & & & & & \\
\hline & pelagicus & e) & e. & 라 & - & -1 & 당 & ?e & 0 & & huxleyi & $\bullet$ & -1 & - & $\bullet$ & 9 & - & - & 바 & & conicus & & & $\bullet$ & & & & & & \\
\hline Coronocyclus & nitiscens & & & - & & - & 0 & e6 & e0 & & ovata & - & $\bullet$ & - & 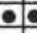 & - & 0 & - & e. & & \begin{tabular}{|l} 
delphix \\
\end{tabular} & & & $\bullet$ & & & & & & \\
\hline Crenalithus & doronicoides & $\bullet$ & • & • & $\bullet$ & $\bullet$ & -6 & 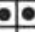 & 6 & Fasciculithus & tympaniformis & & & & & & & & $x$ & & dissimilis & & & $\bullet$ & & & & & $\bullet$ & \\
\hline Cricolithus & jonesii & & & - & - & & & & 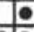 & Gephyrocapsa & caribbeanica & - & $\bullet$ & - & $\bullet$ & e. & 0 & - & e] & & \begin{tabular}{|l|} 
heteromorphus \\
\end{tabular} & & & $\bullet$ & & - & - & - & & 를 \\
\hline Cyclicargolithus & abisectus & & & - & & - & $\bullet$ & 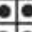 & 0 & & oceanica & - & - & 이 & $\bullet$ & e & 10 & e & 붕 & & moriformis & $x$ & & 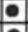 & & - & - & - & & 둔 \\
\hline & floridanus & & & - & & - & e. & e & 0 & & protohuxieyi & & & & & & & & - & & neoabies & $\bullet$ & $\bullet$ & $\bullet$ & $x$ & - & $\bullet$ & - & -1 & ․ \\
\hline Dictyococcites & bisectus & & & - & & & - & - & $x$ & Hayaster & perplexus & - & - & - & - & e. & 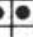 & & & Syracosphaera & pulchra & 0 & $\bullet$ & $\bullet$ & - & - & $\bullet$ & - & - & • \\
\hline & scrippsae & & & • & & & & - & $x$ & Helicosphaera & ampliaperta & & & & & - & & & & Thoracosphaera & heimii & e & $\bullet$ & - & - & - & & & & \\
\hline Discoaster & asymmeiricus & - & - & - & \begin{tabular}{|l|l}
$x$ & \\
\end{tabular} & - & -1e & 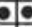 & ( & & Carteri & - & - & - & 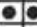 & 0 & 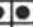 & e & 10 & & saxea & e & - & - & - & - & 0 & - & & 을 \\
\hline & barbadiensis & & & & & & & & $x$ & & euphratis & & - & - & & 0 & - & e & & Triquetro. & carinatus & & & $\bullet$ & & - & & - & $\bullet$ & 리 \\
\hline & bellus & & & - & & - & -1 & - & & & hyalina & - & $\bullet$ & $\bullet$ & - & - & 0 & - & e & mabdulus & milowii & & & - & & & & & & \\
\hline & berggrenii & - & - & - & & - & e. & e? & a & & intermedia & & & & & - & 10 & - & • & 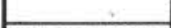 & rugosus & e & - & - & & - & e & - & $\bullet$ & 를 \\
\hline & blackstockae & & -1 & - & & & & & & & recta & & & - & & & & - & & Umbellosphaera & irregularis & e & • & - & - & - & - & - & - & ㄹ. \\
\hline & bollii & & & - & & & & & & & sellii & - & - & - & $\bullet$ & e. & 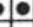 & - & 이 & Umbilicosphaera & cricota & e & - & - & & - & - & - & - & 의 \\
\hline & brouweri & •) & 아 & 인 & - & - & e. & - e & - & & wallichii & • & & & & - & & & & & sibogae & $\bullet$ & $\bullet$ & $\bullet$ & - & - & $\bullet$ & • & $\bullet$ & \\
\hline & calcaris & & & $\bullet$ & & & & & & Heliolithus & Kleinpellii & & & & & & & & $x$ & Watznaueria & barnesae & $x$ & & & & & & & & \\
\hline & challengeri & & $\bullet$ & 다 & & & 5 & e. & & Isthmolithus & recurvus & & & & & & $\bullet$ & & $x$ & Zygrhablithus & bijugatus & & & - & & & & e & & \\
\hline & decorus & $\bullet$ & & & & & & e. & & Lithostromation & perdurum & & & & 10 & e & $\bullet$ & & & & & & & & & & & & & \\
\hline
\end{tabular}

Figure 5. Occurrence plot of all species observed in Quaternary and Neogene sediments at Site 586 (Leg 89) and Sites 587-594 (Leg 90 ). Indigenous species $(-)$ together with reworked species $(\times)$ are listed alphabetically for convenient reference.

\section{Chiasmolithus grandis (Bramlette and Riedel)}

Coccolithus grandis Bramlette and Riedel, 1954, p. 391, pl. 38, figs. la-b.

Chiasmolithus grandis (Bramlette and Riedel). Gartner, 1969b, p. 944, figs. 11-3, 14.

\section{Chiasmolithus oamaruensis (Deflandre)}

Tremalithus oamaruensis Deflandre in Deflandre and Fert, 1954, p. 154 , pl. 11, fig. 22, text-figs. 72-74.

Chiasmolithus oamaruensis (Deflandre). Hay et al., 1966, p. 388, pl. 7 , fig. 1 .

\section{Genus COCCOLITHUS Schwarz, 1894}

Coccolithus carteri (Wallich)

Coccosphaera carteri Wallich, 1877, p. 348, pl. 17, figs. 3, 4, 6, 7, 17. Coccolithus carteri (Wallich). Kamptner, 1941, p. 93.

\section{Coccolithus miopelagicus Bukry}

Coccolithus miopelagicus Bukry, 1971a, p. 310, pl. 2, figs. 6-9.

\section{Coccolithus pelagicus (Wallich)}

Coccosphaera pelagica Wallich, 1877 , p. 348 , pl. 17, figs. 1, 2, 5, 11, 12.

Coccolithus pelagicus (Wallich). Schiller, 1930, p. 246, figs. 123-124.

Genus CORONOCYCLUS Hay, Mohler, and Wade, 1966 Coronocyclus nitescens (Kamptner)

Umbilicosphaera nitescens Kamptner, 1963, pp. 187-188, pl. 1, fig. 5. Coronocyclus nitescens (Kamptner). Bramlette and Wilcoxon, 1967, p. 103, pl. 1, fig. 4; pl. 5, figs. 7-8.
Genus CRENALITHUS Roth, 1973

Crenalithus doronicoides (Black and Barnes)

Coccolithus doronicoides Black and Barnes, 1961, p. 142, pl. 25, fig. 3.

Crenalithus doronicoides (Black and Barnes). Roth, 1973, p. 731, pl. 3 , fig. 3 .

Genus CRICOLITHUS Kamptner, 1958 Cricolithus jonesii Cohen

Cricolithus jonesi Cohen, 1965, p. 16, pl. 2, figs. j, k; pl. 16, figs. a-c.

Genus CYCLICARGOLITHUS Bukry, 1971

Cyclicargolithus abisectus (Müller)

Coccolithus? abisectus Müller, 1970, p. 92, pl. 9, figs. 9, 10; pl. 12, fig. 1.

Cyclicargolithus abisectus (Müller). Bukry, 1973b, p. 703.

Cyclicargolithus floridanus (Roth and Hay)

Coccolithus floridanus Roth and Hay in Hay et al., 1967, p. 445, pl. 6, figs. 1-4.

Cyclicargolithus floridanus (Roth and Hay). Bukry, 1971a, pp. 312-313.

\section{Genus DICTYOCOCCITES Black, 1967}

\section{Dictyococcites bisectus (Hay, Mohler, and Wade)}

Syracosphaera bisecta Hay, Mohler, and Wade, 1966, p. 393, pl. 10, figs. 1-6.

Dictyococcites bisectus (Hay, Mohler, and Wade). Bukry and Percival, 1971, p. 127, pl. 2, figs. 12, 13. 
Dictyococcites scrippsae Bukry and Percival

Dictyococcites scrippsae Bukry and Percival, 1971, p. 128, pl. 2, figs. $7,8$.

Genus DISCOASTER Tan, 1927

Discoaster asymmetricus Gartner

Discoaster asymmetricus Gartner, 1969a, p. 598, pl. 1, figs. 1-3.

Discoaster barbadiensis Tan

Discoaster barbadiensis Tan, 1927, p. 119.

Discoaster bellus Bukry and Percival

Discoaster bellus Bukry and Percival, 1971, p. 128, pl. 3, figs. 1, 2.

Discoaster berggrenii Bukry

Discoaster berggrenii Bukry, 1971b, p. 45, pl. 2, figs. 4-6.

Discoaster blackstockae Bukry

Discoaster blackstockae Bukry, 1973c, p. 307, pl. 1, figs. 1-4.

Discoaster bollii Martini and Bramlette

Discoaster bollii Martini and Bramlette, 1963, p. 851, pl. 105, figs. 1$4,7$.

\section{Discoaster brouweri Tan}

Discoaster brouweri Tan, 1927 , p. 120, figs. 8 a-b.

\section{Discoaster calcaris Gartner}

Discoaster calcaris Gartner, 1967, p. 2, pl. 2, figs. 1-3.

\section{Discoaster challengeri Bramlette and Riedel}

Discoaster challengeri Bramlette and Riedel, 1954, p. 401, pl. 39, fig. 10.

\section{Discoaster decorus (Bukry)}

Discoaster variabilis decorus Bukry, 1971b, p. 48, pl. 3, figs. 5, 6. Discoaster decorus (Bukry). Bukry, 1973a, p. 677, pl. 2, figs. 8, 9; pl. 4, fig. 11.

\section{Discoaster deflandrei Bramlette and Riedel}

Discoaster deflandrei Bramlette and Riedel, 1954, p. 399, pl. 39, fig. 6; text-figs. 1a-c.

\section{Discoaster druggii Bramlette and Wilcoxon}

Discoaster druggii Bramlette and Wilcoxon, 1967, p. 220 (nom. subst. pro D. extensus Bramlette and Wilcoxon, 1967, non Hay, 1967).

\section{Discoaster exilis Martini and Bramlette}

Discoaster exilis Martini and Bramlette, 1963, p. 852, pl. 104, figs. 1-3.

\section{Discoaster hamatus Martini and Bramlette}

Discoaster hamatus Martini and Bramlette, 1963, p. 852, pl. 105, figs. $8,10,11$.

\section{Discoaster intercalaris Bukry}

Discoaster intercalaris Bukry, 1971a, p. 315, pl. 3, fig. 12; pl. 4, figs. $1,2$.

Discoaster kugleri Martini and Bramlette

Discoaster kugleri Martini and Bramlette, 1963, p. 853, pl. 102, figs. 11-13.

Discoaster lodoensis Bramlette and Riedel

Discoaster lodoensis Bramlette and Riedel, 1954, p. 398, pl. 39, figs. 3a-b.
Discoaster loeblichii Bukry

Discoaster loeblichii Bukry, 1971a, pp. 315-316, pl. 4, figs. 3-5.

\section{Discoaster moorei Bukry}

Discoaster moorei Bukry, 1971b, p. 46, pl. 2, figs. 11, 12; pl. 3, figs. 1,2 .

Discoaster neohamatus Bukry and Bramlette

Discoaster neohamatus Bukry and Bramlette, 1969, p. 133, pl. 1, figs. 4-6.

Discoaster pentaradiatus Tan

Discoaster pentaradiatus Tan, 1927, p. 120, fig. 2.

Discoaster perclarus Hay

Discoaster perclarus Hay in Hay et al., 1967, p. 452, pl. 4, figs. 11, 12.

\section{Discoaster quinqueramus Gartner}

Discoaster quinqueramus Gartner, 1969a, p. 598, pl. 1, figs. 6, 7.

Discoaster stellulus Gartner

Discoaster stellulus Gartner, 1967, p. 3, pl. 4, figs. 1-3.

Discoaster surculus Martini and Bramlette

Discoaster surculus Martini and Bramlette, 1963, p. 854, pl. 104, figs. $10-12$.

\section{Discoaster tamalis Kamptner}

Discoaster tamalis Kamptner, 1967, p. 166, pl. 24, fig. 131; text-fig. 28.

\section{Discoaster tridenus Kamptner}

Discoaster tridenus Kamptner, 1967, p. 166, text-fig. 30.

\section{Discoaster triradiatus Tan}

Discoaster triradiatus Tan, 1927, p. 417.

\section{Discoaster variabilis Martini and Bramlette}

Discoaster variabilis Martini and Bramlette, 1963, p. 854, pl. 104, figs. 4-8.

\section{Genus DISCOLITHINA Loeblich and Tappan, 1963} Discolithina japonica Takayama

Discolithina japonica Takayama, 1967, p. 177, 181, 189, pl. 2, fig. 11; pl. 9; pl. 10, text-figs. 6, 7.

\section{Discolithina multipora (Kamptner)}

Discolithus multiporus Kamptner, 1948, p. 5, pl. 1, fig. 9.

Discolithina multipora (Kamptner). Martini, 1965, p. 400.

\section{Genus DISCOSPHAERA Haeckel, 1894}

Discosphaera tubifera (Murray and Blackman)

Rhabdosphaera tubifer Murray and Blackman, 1898, p. 438, pl. 15, figs. 8-11.

Discosphaera tubifera (Murray and Blackman). Ostenfeld, 1900, p. 200.

\section{Genus EMILIANIA Hay and Mohler, 1967}

Emiliania annula (Cohen)

Coccolithites annulus Cohen, 1964, p. 237, pl. 3, figs. 1a-c.

Emiliania annula (Cohen). Bukry, 1973a, p. 678.

\section{Emiliania huxleyi (Lohmann)}

Pontosphaera huxleyi Lohmann, 1902, p. 130, pl. 4, figs. 1-6; pl. 6, fig. 69.

Emiliania huxleyi (Lohmann). Hay and Mohler in Hay et al., 1967, p. 447 , pl. 10, 11, figs. 1, 2. 


\section{Emiliania ovata Bukry}

Emiliania ovata Bukry, 1973a, p. 678, pl. 2, figs. 10-12.

Genus FASCICULITHUS Bramlette and Sullivan, 1961

Fasciculithus tympaniformis Hay and Mohler

Fasciculithus tympaniformis Hay and Mohler in Hay et al., 1967, p. 447 , pl. 8, figs. 1-5; pl. 9, figs. 1-5.

Genus GEPHYROCAPSA Kamptner, 1943

Gephyrocapsa caribbeanica Boudreaux and Hay

Gephyrocapsa caribbeanica Boudreaux and Hay in Hay et al., 1967, p. 447 , pl. 12,13 , figs. 1-4.

Gephyrocapsa oceanica Kamptner

Gephyrocapsa oceanica Kamptner, 1943, pp. 43-49.

Gephyrocapsa protohuxleyi McIntyre

Gephyrocapsa protohuxley McIntyre, 1970, pp. 187-189, fig. 1a-g.

Genus HAYASTER Bukry, 1973

Hayaster perplexus (Bramlette and Riedel)

Discoaster perplexus Bramlette and Riedel, 1954, p. 400, pl. 39, fig. 9. Hayaster perplexus (Bramlette and Riedel). Bukry, 1973c, p. 308.

Genus HELICOSPHAERA Kamptner, 1954

Helicosphaera ampliaperta (Bramlette and Wilcoxon)

Helicopontosphaera ampliaperta Bramlette and Wilcoxon, 1967, p. 105 , pl. 6 , figs. $1-4$.

Helicosphaera ampliaperta (Bramlette and Wilcoxon). Jafar and Martini, 1975 , p. 390.

Helicosphaera carteri (Wallich)

Helicosphaera carteri (Wallich). Kamptner, 1954, pp. 21-23, 73-74, text-figs. 17a-c, 18, 19. Jafar and Martini, 1975, pp. 381-397, pl. 1 , figs. $1,4,5$.

Helicopontosphaera kamptneri Hay and Mohler in Hay et al., 1967, p. 448 , pl. 10 , fig. 5 ; pl. 11 , fig. 5 .

\section{Helicosphaera euphratis Haq}

Helicosphaera euphratis Haq, 1966, p. 33, pl. 2, figs. 1, 3.

Helicopontosphaera euphratis (Haq). Martini, 1969, p. 136.

\section{Helicosphaera hyalina Gaarder}

Helicosphaera hyalina Gaarder, 1970, pp. 113-114, text-figs. 1-3.

Helicopontosphaera hyalina (Gaarder). Haq, 1973, p. 37.

\section{Helicosphaera intermedia Martini}

Helicosphaera intermedia Martini, 1965, p. 404, pl. 35, figs. 1, 2.

Helicopontosphaera intermedia (Martini). Hay and Mohler in Hay et al., 1967, p. 448.

Helicosphaera recta (Haq)

Helicosphaera seminulum recta Haq, 1966, p. 34, pl. 2, fig. 6; pl. 3, fig. 4.

Helicopontosphaera recta (Haq). Martini, 1969, p. 136.

Helicosphaera recta (Haq). Jafar and Martini, 1975, p. 391.

\section{Helicosphaera sellii (Bukry and Bramlette)}

Helicopontosphaera sellii Bukry and Bramlette, 1969, p. 134, pl. 2, figs. 3-7.

Helicosphaera sellii (Bukry and Bramlette). Jafar and Martini, 1975, p. 391; Ellis and Lohman, 1979, p. 76.

Helicosphaera inversa Gartner, 1977, p. 23, pl. 1, figs. 4a-b, 5a-c.

\section{Helicosphaera wallichii (Lohmann)}

Coccosphaera wallichi Lohmann, 1902, p. 138, pl. 5, figs. 58-60.

Helicopontosphaera wallichi (Lohmann). Boudreaux and Hay, 1969, pp. 272-273, pl. 6, fig. 9.
Helicosphaera wallichii (Lohmann). Okada and McIntyre, 1977, pp. $14-15$, pl. 4 , fig. 8.

Genus HELIOLITHUS Bramlette and Sullivan, 1961

Heliolithus kleinpellii Sullivan

Heliolithus kleinpellii Sullivan, 1964, p. 193, pl. 12, fig. 5.

\section{Genus ISTHMOLITHUS Deflandre, 1954}

Isthmolithus recurvus Deflandre

Isthmolithus recurvus Deflandre in Deflandre and Fert, 1954, p. 169, pl. 12, figs. 9-13, text-figs. 119-122.

\section{Genus LITHOSTROMATION Deflandre, 1942 \\ Lithostromation perdurum Deflandre}

Lithostromation perdurum Deflandre, 1942b, p. 918, figs. 1-9.

Genus LOPHODOLITHUS Deflandre, 1954

Lophodolithus nascens Bramlette and Sullivan

Lophodolithus nascens Bramlette and Sullivan, 1961, p. 145, pl. 4, figs. $7 \mathrm{a}-\mathrm{c}, 8 \mathrm{a}-\mathrm{c}$.

Genus OOLITHOTUS Reinhardt, in Cohen and Reinhardt, 1968 Oolithotus fragilis (Lohmann)

Coccolithophora fragilis Lohmann, 1912, pp. 49, 54, text-fig. 11. Discolithus antillarum Cohen, 1964, p. 236, pl. 1, figs. 3a-e.

Oolithotus fragilis (Lohmann). Okada and McIntyre, 1977, p. 11, pl. 4, fig. 3.

Genus ORTHORHABDUS Bramlette and Wilcoxon, 1967

Orthorhabdus serratus Bramlette and Wilcoxon

Orthorhabdus serratus Bramlette and Wilcoxon, 1967, pp. 114-116, pl. 9, figs. 5-10.

Genus PONTOSPHAERA Lohmann, 1902 Pontosphaera discopora Schiller

Pontosphaera discopora Schiller, 1925, p. 11, pl. 1, fig. 4.

Genus RETICULOFENESTRA Hay, Mohler, and Wade, 1966

Reticulofenestra hillae Bukry and Percival

Reticulofenestra hillae Bukry and Percival, 1971, p. 136, pl. 6, figs. 1-3.

Reticulofenestra pseudoumbilica (Gartner)

Coccolithus pseudoumbilicus Gartner, 1967, p. 4, pl. 6, fig. 3.

Reticulofenestra pseudoumbilica (Gartner). Gartner, 1969a, pp. 587589.

Reticulofenestra umbilica (Levin)

Coccolithus umbilicus Levin, 1965, p. 265, pl. 41, fig. 2.

Reticulofenestra umbilica (Levin). Martini and Ritzkowski, 1968, p. 245 , pl. 1, figs. 11,12 .

\section{Genus RHABDOSPHAERA Haeckel, 1894}

Rhabdosphaera clavigera Murray and Blackman

Rhabdosphaera clavigera Murray and Blackman, 1898, p. 438, pl. 15, figs. 13-15.

Rhabdosphaera procera Martini

Rhabdosphaera procera Martini, 1969, p. 289, pl. 26, figs. 10, 11.

Genus SCAPHOLITHUS Deflandre, 1954

Scapholithus fossilis Deflandre

Scapholithus fossilis Deflandre in Deflandre and Fert, 1954, p. 165, pl. 8 , figs. $12,16,17$. 
Genus SCYPHOSPHAERA Lohmann, 1902

Scyphosphaera amphora Deflandre

Scyphosphaera amphora Deflandre, 1942a, p. 132, figs. 21, 22.

Scyphosphaera apsteinii Lohmann

Scyphosphaera apsteinii Lohmann, 1902, p. 132, pl. 4, figs. 26-30.

Scyphosphaera campanula Deflandre

Scyphosphaera campanula Deflandre, 1942a, pl. 134, figs. 23-27.

Scyphosphaera conica Kamptner

Scyphosphaera conica Kamptner, 1955, p. 26, figs. 130, 131.

Scyphosphaera cylindrica Kamptner

Scyphosphaera cylindrica Kamptner, 1955, p. 24, fig. 119.

Scyphosphaera globulata Bukry and Percival

Scyphosphaera globulata Bukry and Percival, 1971, pp. 138-140, pl. 7, figs. 1-6.

\section{Scyphosphaera intermedia Deflandre}

Scyphosphaera intermedia Deflandre, 1942a, p. 134, figs. 32-36.

\section{Scyphosphaera magna Kamptner}

Scyphosphaera magna Kamptner, 1967, p. 150, text-figs. 20.

Scyphosphaera pulcherrima Deflandre

Scyphosphaera pulcherrima Deflandre, 1942a, p. 133, figs. 28-31.

Scyphosphaera recurvata Deflandre

Scyphosphaera recurvata Deflandre, 1942a, p. 132, figs. 17-20.

\section{Genus SPHENOLITHUS Deflandre, 1952}

Sphenolithus abies Deflandre

Sphenolithus abies Deflandre in Deflandre and Fert, 1954, p. 164, pl. 10 , figs. 1-4.

Sphenolithus belemnos Bramlette and Wilcoxon

Sphenolithus belemnos Bramlette and Wilcoxon, 1967, p. 118, pl. 2, figs. 1-3.

Sphenolithus capricornutus Bukry and Percival

Sphenolithus capricornutus Bukry and Percival, 1971, p. 140, pl. 6, figs. 4-6.

Sphenolithus conicus Bukry

Sphenolithus conicus Bukry, 1971a, p. 320, pl. 5, figs. 10-12.

Sphenolithus delphix Bukry

Sphenolithus delphix Bukry, 1973a, p. 679, pl. 3, figs. 19-22.

Sphenolithus dissimilis Bukry and Percival

Sphenolithus dissimilis Bukry and Percival, 1971, p. 140, pl. 6, figs. 7-9.

Sphenolithus heteromorphus Deflandre

Sphenolithus heteromorphus Deflandre, 1953, pp. 1785-1786, figs. $1,2$.

Sphenolithus moriformis (Brönnimann and Stradner)

Nannoturbella moriformis Brönnimann and Stradner, 1960, p. 368 , figs. 11-16.

Sphenolithus moriformis (Brönniman and Stradner). Bramlette and Wilcoxon, 1967, pp. 124-126, pl. 3, figs. 1-6.

Sphenolithus neoabies Bukry and Bramlette

Sphenolithus neoabies Bukry and Bramlette, 1969, p. 140, pl. 3, figs. 9-11.
Genus SYRACOSPHAERA Lohmann, 1902

Syracosphaera pulchra Lohmann

Syracosphaera pulchra Lohmann, 1902, p. 134, pl. 4, figs. 33, 36, 37.

Genus THORACOSPHAERA Kamptner, 1927

Thoracosphaera heimii (Lohmann)

Syracosphaera heimii Lohmann, 1919, p. 117, fig. 29.

Thoracosphaera heimii (Lohmann). Kamptner, 1954, pp. 40-42, figs. $41,42$.

Thoracosphaera saxea Stradner

Thoracosphaera saxea Stradner, 1961, p. 84, fig. 71.

Genus TRIQUETRORHABDULUS Martini, 1965

Triquetrorhabdulus carinatus Martini

Triquetrorhabdulus carinatus Martini, 1965, p. 408, pl. 36, figs. 1-3.

Triquetrorhabdulus milowii Bukry

Triquetrorhabdulus milowii Bukry, 1971a, p. 325, pl. 7, figs. 9-12.

Triquetrorhabdulus rugosus Bramlette and Wilcoxon

Triquetrorhabdulus rugosus Bramlette and Wilcoxon, 1967, pp. 128129 , pl. 9 , figs. 17,18 .

Genus UMBELLOSPHAERA Paasche, 1955

Umbellosphaera irregularis Paasche

Umbellosphaera irregularis Paasche in Markali and Paasche, 1955, p. 97, pl. 3-6.

\section{Genus UMBILICOSPHAERA Lohmann, 1902}

Umbilicosphaera cricota (Gartner)

Cyclococcolithus cricotus Gartner, 1967, p. 5, pl. 7, figs. 5-7.

Umbilicosphaera cricota (Gartner). Cohen and Reinhardt, 1968, p. 296, pl. 19, figs. 1, 5; pl. 21, fig. 3; text-fig. 6 .

Umbilicosphaera sibogae (Weber-van Bosse)

Coccosphaera sibogae Weber-van Bosse, 1901, p. 137, 140, pl. 17, figs. $1,2$.

Umbilicosphaera mirabilis Lohmann, 1902, p. 139, pl. 5, figs. 66, 66 .

Umbilicosphaera sibogae (Weber-van Bosse). Gaarder, 1970, p. 126.

Genus WATZNAUERIA Reinhardt, 1964

Watznaueria barnesae (Black)

Tremalithus barnesae Black in Black and Barnes, 1959, p. 325, pl. 9, figs. $1,2$.

Watznaueria barnesae (Black). Perch-Nielsen, 1968, p. 69, pl. 22, figs. $1-7$; pl. 23, figs. $1,4,5,16$; text-fig. 32 .

Genus ZYGRHABLITHUS Deflandre, 1959

Zygrhablithus bijugatus (Deflandre)

Zygolithus bijugatus Deflandre in Deflandre and Fert, 1954, p. 148, pl. 11, figs. 20,21 .

Zygrhablithus bijugatus (Deflandre). Deflandre, 1959, p. 135.

\section{SUMMARY OF NANNOPLANKTON BIOSTRATIGRAPHY}

The nannoplankton biostratigraphy is summarized for each of the nine sites, together with brief site descrip. tions. The geologic age and nannoplankton zone or subzone assignment of the Quaternary and Neogene cores from Hole 586B (Leg 89) and the holes from Sites 587594 (Leg 90) are shown in Table 1. In addition, a table of nannoplankton occurrences for each site has been prepared (Tables 2-10, later). For some sites where multiple holes were drilled, sets of samples from more than one 
Table 1. Geologic age and nannoplankton zone assignment of Quaternary and Neogene cores from Site 586 (Leg 89) and Sites 587-594 (Leg 90).

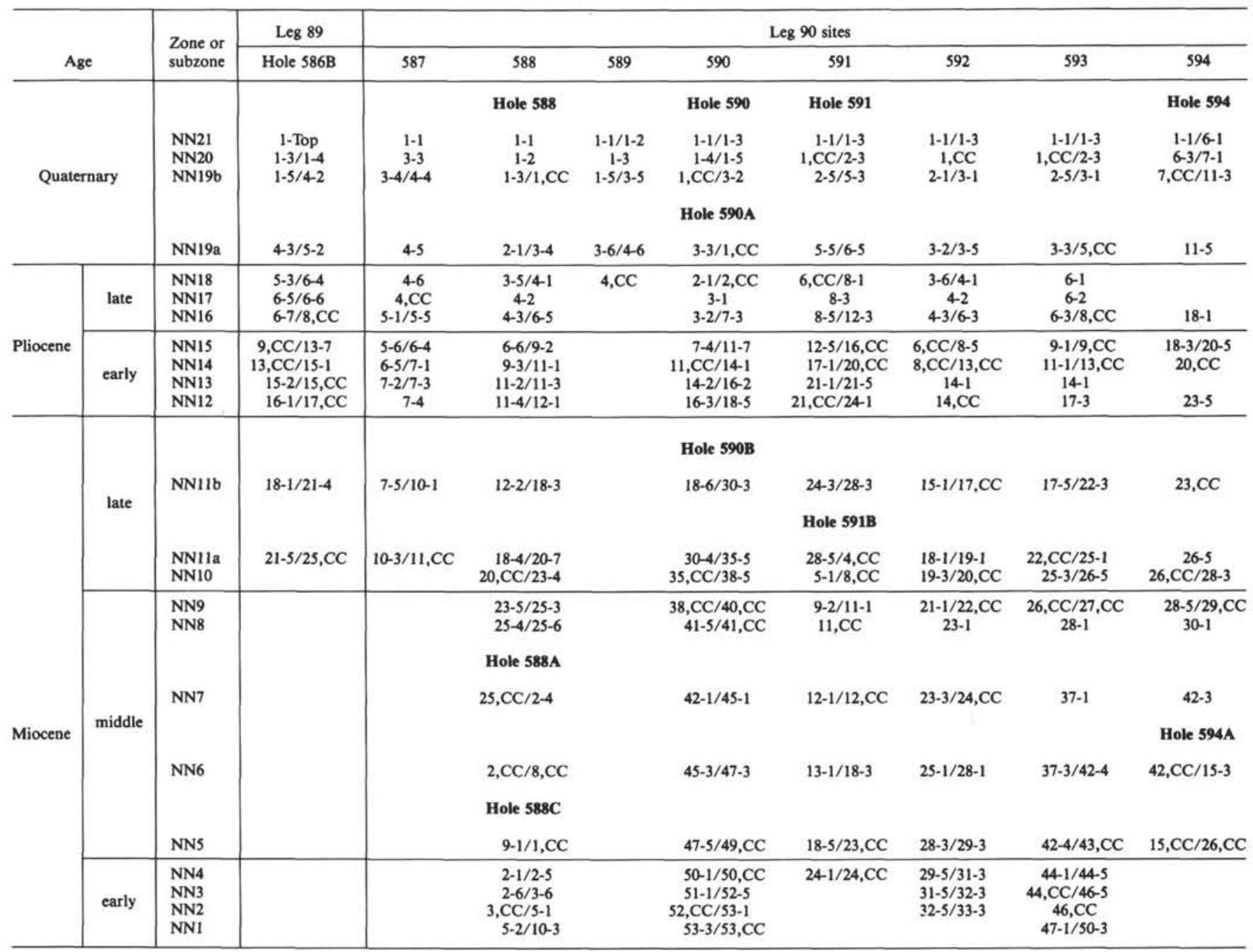

Note: Slash separates first section of zonal interval from last section of interval.

hole were examined in order to provide complete stratigraphic coverage. For convenient reference, the index species are grouped together in the tables and the additional species are then listed alphabetically. The state of preservation is designated as $\mathrm{G}=$ good, little or no etching or overgrowth; $\mathrm{F}$ = fair, some etching or overgrowth which has damaged or obscured ornamentation; $\mathrm{P}=$ poor, considerable etching or overgrowth which has made many species difficult to recognize. Abundance values of each taxon were obtained by scanning an area measuring approximately $45 \mathrm{~mm}^{2}$ and assigning the following values: $A=$ abundant ( $>50$ specimens), $\mathrm{C}=$ common (25-49 specimens), and $\mathrm{R}=$ rare $(<25$ specimens). Over 700 samples from the nine sites were examined with a light microscope to determine the Neogene and Quaternary nannoplankton biostratigraphy. In addition, 61 samples were further examined with a scanning electron microscope (SEM) to determine the range of Emiliania huxleyi. Time did not permit further SEM examination to determine the presence of other small Quaternary species.

All relevant zonal and subzonal indicators are present at Sites 586-591. However, at sites south of Site 591, some indicators become increasingly rare and consequently unusable, or they are absent. The paucity of Ceratolithus rugosus at Site 592 and its absence at Sites 593 and 594 prevents recognition of the NN13/NN12 boundary at these sites and the absence of Catinaster coalitus at Sites 593 and 594 prevents recognition of the NN8/ NN7 boundary. At Site 594, $40 \%$ of the zonal and subzonal indicators are either missing or occur too sparsely to use. In addition to the absence of Ceratolithus rugosus and Catinaster coalitus, the absence of Amaurolithus primus and the sparse occurrences of Discoaster brouweri, $D$. pentaradiatus, $D$. surculus, and $D$. asymmetricus prevent recognition of the NN19/NN18, NN18/NN17, NN17/NN16, NN14/NN13, and NN11b/NN11a zonal and subzonal boundaries.

\section{Site $\mathbf{5 8 6}$}

\section{Hole 586B}

Site 586 is located on the northeastern upper slope of the Ontong-Java Plateau $\left(00^{\circ} 29.84^{\prime} \mathrm{S}, 158^{\circ} 29.89^{\prime} \mathrm{E}\right)$ in tropical waters at a depth of $2207 \mathrm{~m}$ below sea level. Four holes were drilled at this site during DSDP Leg 89. 
The 25 cores obtained from Hole 586B were set aside for study by DSDP Leg 90 scientists in order to complete the latitudinal traverse. Hole 586B was terminated at a depth of $240.3 \mathrm{~m}$ because equipment failed.

All of the zonal and subzonal indicators for Zones NN21 through NN11 are present and species diversity is good throughout Hole 586B (Table 2). Preservation changes from good to fair below Sample 586B-6-2, 30$31 \mathrm{~cm}$, and only minor reworking was encountered.

\section{Quaternary}

The Quaternary includes Cores 1-4 and the upper part of Core 5 above the last occurrence of Discoaster brouweri in Sample 586B-5-3, 30-31 cm. Two rare occurrences of $D$. brouweri above this sample are reworked. The rare occurrence of Emiliania huxleyi in Sample 586B-1, top, places the uppermost part of Core 1 in Zone NN21. The two underlying samples (586B-1-3, 30-31 cm and 586B$1-4,30-31 \mathrm{~cm}$ ) belong in Zone NN20. The last occurrences of $E$. ovata in Sample 586B-1-5, 30-31 cm and Calcidiscus macintyrei in Sample 586B-4-3, 30-31 cm place Samples 586B-1-5, 30-31 cm through 586B-4-2, 30-31 cm in Subzone NN19b and Samples 586B-4-3, 30$31 \mathrm{~cm}$ through 586B-5-2, 30-31 cm in Subzone NN19a.

\section{Pliocene}

The Pliocene includes the lower part of Core 5 and Cores 6-17. The last occurrence of Reticulofenestra pseudoumbilica in Sample 586-9,CC marks the lower/upper Pliocene boundary. The last occurrences of Discoaster pentaradiatus in Sample 586B-6-5, 30-31 cm and D. surculus in Sample 586B-6-7, 30-31 cm place Samples 586B$5-3,30-31 \mathrm{~cm}$ through 586B-6-4, 30-31 cm in Zone NN18, Samples 586B-6-5, 30-31 cm and 586B-6-6, 30$31 \mathrm{~cm}$ in Zone NN17, and Sample 586B-6-7, 30-31 cm through Core 8 in Zone NN16. The last occurrence of Amaurolithus tricorniculatus in Sample 586B-13,CC places Samples 586B-9,CC through 586B-13-7, 30-31 $\mathrm{cm}$ in Zone NN15. Zone NN14 includes Sample 586B$13, \mathrm{CC}$ to the first consistent occurrence of $D$. asymmetricus in Sample 586B-15-1, 30-31 cm. The numerous occurrences of $D$. asymmetricus below this sample are probably due to contamination. The first occurrence of Ceratolithus rugosus in Sample 586B-15,CC places Samples 586B-15-2, 30-31 cm through 586B-15,CC in Zone NN13 and Samples 586B-16-1, 30-31 cm through 586B$17, \mathrm{CC}$ in Zone NN12.

\section{Miocene}

The occurrence of Discoaster quinqueramus in Sample 586B-18-1, 30-31 cm through Core 25 determined that Hole 586B terminated within the late Miocene Zone NN11. This zone is subdivided into Subzones NN11b and NN11a by the first occurrence of Amaurolithus primus in Sample 586B-21-4, 30-31 cm.

\section{Site $\mathbf{5 8 7}$}

\section{Hole 587}

Site 587 is located on the southern slope of Lansdowne Bank $\left(21^{\circ} 11.87^{\prime} \mathrm{S}, 161^{\circ} 19.99^{\prime} \mathrm{E}\right)$ in subtropical waters at a depth of $1101 \mathrm{~m}$ below sea level. One hole was cored to a depth of $147.0 \mathrm{~m}$ and 11 cores were obtained with good recovery. A sudden change in lithology at a depth of $99.1 \mathrm{~m}$ prevented further core recovery.

With the exception of the NN21/NN20 boundary, all of the nannoplankton zones and subzones through Subzone NN11a are recognized (Table 3 ). Species diversity is good throughout the hole. Preservation is good through Core 4, fair in Cores 5-10, and poor in Core 11. Rare reworked discoasters were observed in three of the Quaternary samples.

\section{Quaternary}

The Quaternary includes Cores 1-3 and most of Core 4. Rare to common occurrences of Emiliania huxleyi in all of the samples above the last occurrence of $E$. ovata in Sample 587-3-4, 20-21 cm prevent recognition of the boundary between Zones NN21 and NN20. Samples 587$1-1,4-5 \mathrm{~cm}$ through 587-3-3, 20-21 cm are therefore placed in the combined Zone NN21/NN20. A thin Zone NN20 may, however, occur between Samples 587-3-3, $20-21 \mathrm{~cm}$ and $587-3-4,20-21 \mathrm{~cm}$. The last occurrence of Discoaster brouweri in Sample 587-4-6, 4-5 cm places Samples 587-3-4, 20-21 cm through 587-4-5, 4-5 cm in Zone NN19. The last occurrence of Calcidiscus macintyrei in Sample 587-4-5, 4-5 cm subdivides Zone NN19 into Subzones NN19b and NN19a.

\section{Pliocene}

The Pliocene includes the lower part of Core 4 through the upper part of Core 7. The last occurrence of Reticulofenestra pseudoumbilica in Sample 587-5-6, 4-5 cm marks the lower/upper Pliocene boundary. The presence of Discoaster brouweri and the absence of $D$. pentaradiatus in Sample 587-4-6, 4-5 cm places this sample in Zone NN18. The presence of $D$. pentaradiatus and the absence of $D$. surculus in Sample 587-4,CC places this sample in Zone NN17. The interval from the last occurrence of D. surculus down to Sample 587-5-5, 4-5 $\mathrm{cm}$, above the last occurrence of Reticulofenestra pseudoumbilica in Sample 587-5-6, 4-5 cm, belongs in Zone NN16. Zone NN15 includes Samples 587-5-6, 4-5 cm through 587-6-4, 4-5 cm. The last occurrence of Amaurolithus tricorniculatus in Sample 587-6-5, 4-5 cm and the first occurrence of $D$. asymmetricus in Sample 587. $7-1,4-5 \mathrm{~cm}$ place this interval in Zone NN14. The first consistent occurrence of Ceratolithus rugosus in Sample 587-7-3, 4-5 cm places this sample and the overlying Sample 587-7-2, 4-5 cm in Zone NN13. Sample 587-7-4, $4-5 \mathrm{~cm}$ belongs in Zone NN12.

\section{Miocene}

The last occurrence of Discoaster quinqueramus in Sample 587-7-5, 4-5 cm and the first occurrence of Amaurolithus primus in Sample 587-10-1, 12-13 cm place this interval in Subzone NN11b. Sample 587-10-3, 12-13 cm through $587-11-1,20-21 \mathrm{~cm}$ contain $D$. quinqueramus without $A$. primus and therefore belong in Subzone NN11a. Because preservation is poor and zonal indicators are missing below Sample 587-11-1, 20-21 cm, it can only be assumed that the remaining Core 11 samples also belong in Subzone NN11a. 
Table 2. Nannoplankton occurrences, Hole 586B.



Site $\mathbf{5 8 8}$

Holes 588, 588A, 588C

Site 588 is located on the northern Lord Howe Rise $\left(26^{\circ} 06.70^{\prime} \mathrm{S}, 161^{\circ} 13.60^{\prime} \mathrm{E}\right)$ in subtropical waters at a depth of $1533 \mathrm{~m}$ below sea level. Four holes were drilled at this site. Hole 588 was cored to a depth of $236.0 \mathrm{~m}$ and 25 cores were obtained. Hole 588A was washed down to a depth of $236.0 \mathrm{~m}$ and then cored to a depth of $344.4 \mathrm{~m}$. Eighteen cores were taken. Hole 588B, which duplicates Hole 588, was cored to a depth of $277.4 \mathrm{~m}$ and 31 cores were taken. Hole $588 \mathrm{C}$ was washed down to a depth of $305.7 \mathrm{~m}$ and then cored to a depth of 488.1 m. Nineteen cores were obtained. Core recovery was generally good throughout the four holes, but recovery was poor in the lowest sections of Holes 588A and $588 \mathrm{C}$.

In order to obtain a complete stratigraphic section, cores from three holes $(588,588 \mathrm{~A}$, and $588 \mathrm{C})$ were examined for calcareous nannoplankton (Table 4). Species diversity is good throughout the holes. Preservation changes from good to fair below Core 7 of Hole 588 and only minor reworking was encountered. The major regional unconformity of the southwest Pacific was encountered within Core 18 in the lower part of Hole 588C. It separates middle Eocene sediments (NP15/ 
Table 3. Nannoplankton occurrences, Site 587.

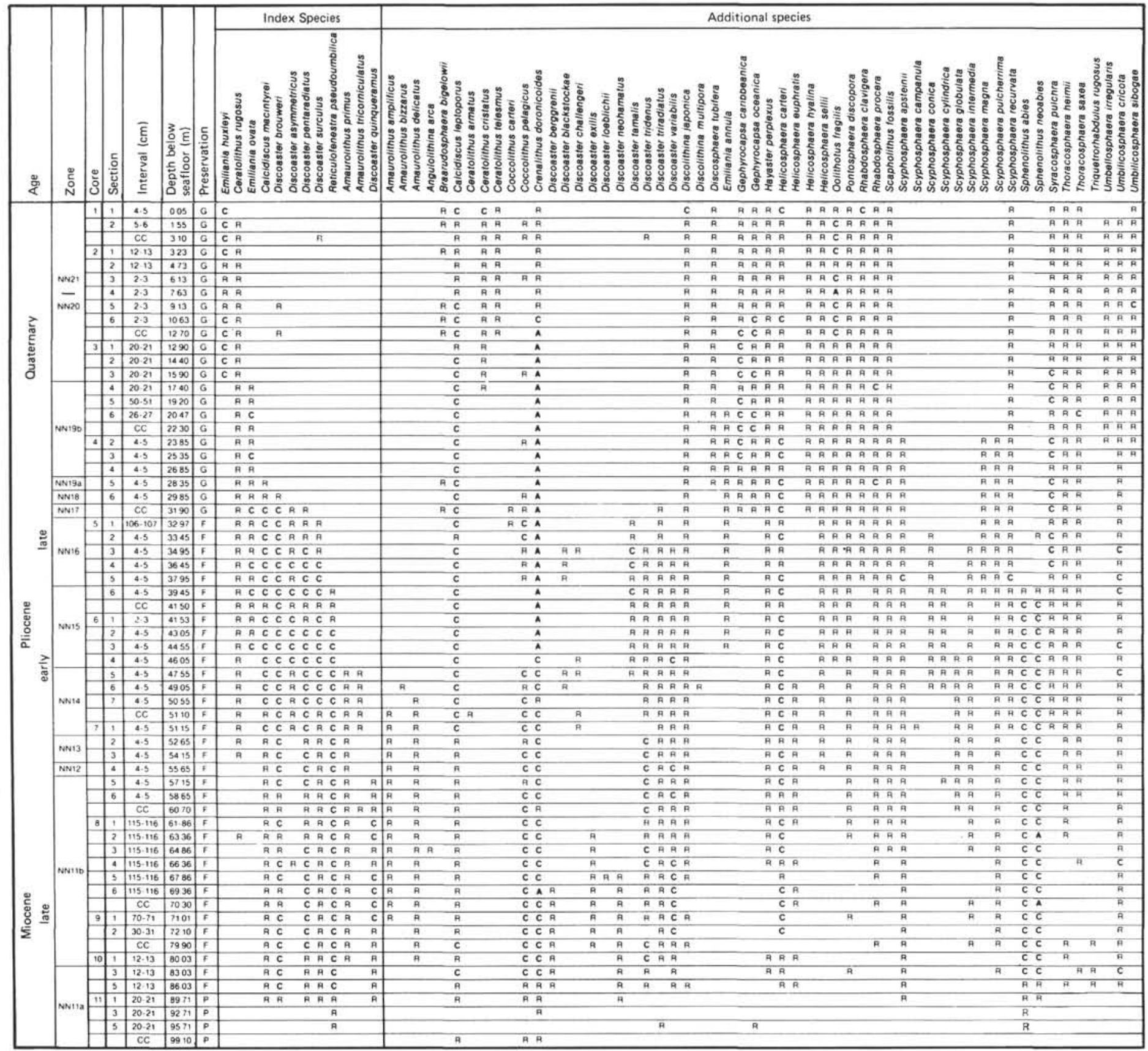


Table 4. Nannoplankton occurrences, Holes 588, 588A, and 588C.

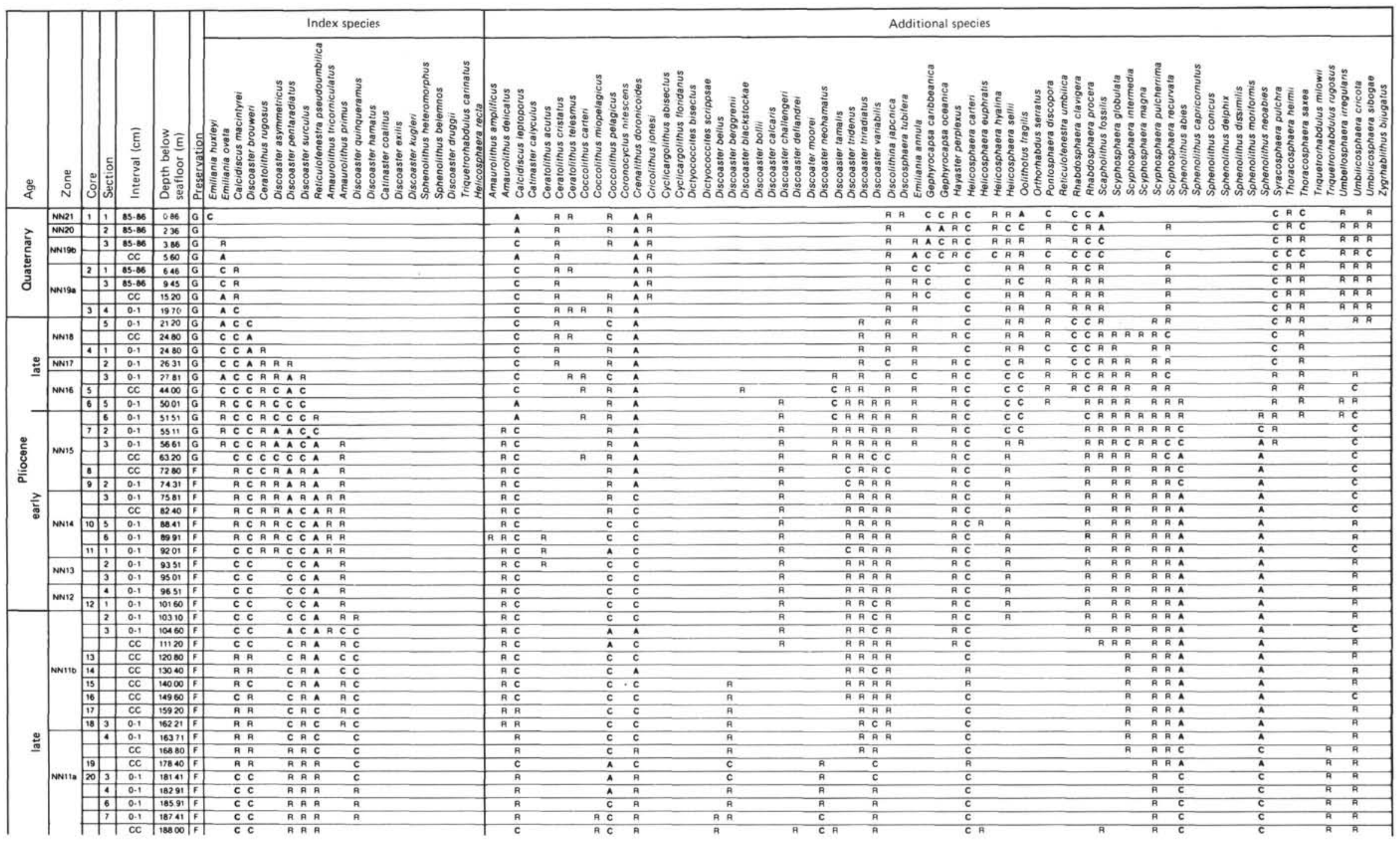




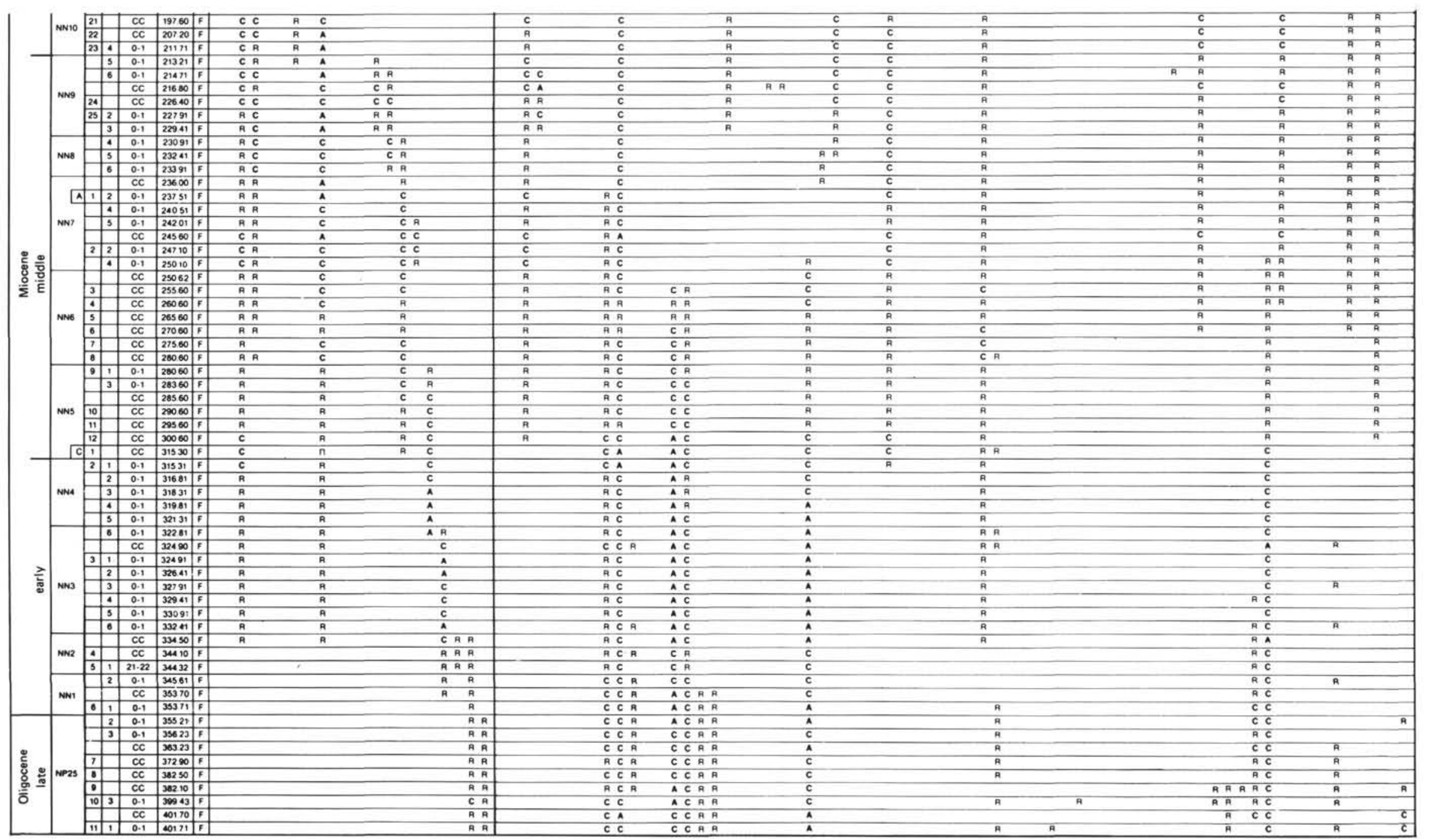


NP16) from immediately overlying upper Oligocene sediments (NP24). A complete Quaternary, Neogene, and upper Oligocene section overlies the unconformity.

\section{Quaternary}

The Quaternary includes Cores 1-2 and the upper part of Core 3. The presence of Emiliania huxleyi in Sample 588-1-1, 85-86 cm places this sample in Zone NN21. The last occurrence of E. ovata in Sample 588$1-3,85-86 \mathrm{~cm}$ places the intervening Sample 588-1-2, $85-86 \mathrm{~cm}$ in Zone NN20. The last occurrence of Discoaster brouweri in Sample 588-3-5, 0-1 cm places Samples $588-1-3,85-86 \mathrm{~cm}$ through $588-3-4,0-1 \mathrm{~cm}$ in Zone NN19. The last occurrence of Calcidiscus macintyrei in Sample 588-2-1, 85-86 cm subdivides Zone NN19 into Subzones NN19b and NN19a.

\section{Pliocene}

The Pliocene section includes the lower part of Core 588-3 through the upper part of Core 588-12. The last occurrence of Reticulofenestra pseudoumbilica in Sample 588-6-6, 0-1 cm marks the lower/upper Pliocene boundary. The presence of Discoaster brouweri and the absence of $D$. pentaradiatus in Samples 588-3-5, 0-1 cm through 588-4-1, 0-1 cm place these samples in Zone NN18. Sample 588-4-2, 0-1 cm contains D. pentaradiatus without $D$. surculus and belongs in Zone NN17. The interval from the last occurrence of $D$. surculus in Sample 588-4-3, 0-1 cm through Sample 588-6-5, 0-1 cm is placed in Zone NN16. The last occurrences of $R$. pseudoumbilica in Sample 588-6-6, 0-1 cm and Amaurolithus tricorniculatus in Sample 588-9-3, 0-1 cm place Samples 588-6-6, 0-1 cm through 588-9-2, 0-1 cm in Zone NN15. The first occurrences of D. asymmetricus in Sample 588-11-1, 0-1 cm and Ceratolithus rugosus in Sample $588-11-3,0-1 \mathrm{~cm}$ place Samples $588-9-3,0-1 \mathrm{~cm}$ through 588-11-1, 0-1 cm in Zone NN14 and Samples $588-11-2,0-1 \mathrm{~cm}$ and $588-11-3,0-1 \mathrm{~cm}$ in Zone NN13. Samples 588-11-4, 0-1 cm and 588-12-1, 0-1 cm, between the first occurrence of $C$. rugosus and the last occurrence of $D$. quinqueramus, belong in Zone NN12.

\section{Miocene}

The Miocene includes the upper part of Core 12, Hole 588 , through the upper part of Core 10 , Hole 588C. The middle/upper Miocene boundary lies between Samples 588-23-4, 0-1 cm and 588-23-5, 0-1 cm and the lower/ middle Miocene boundary lies between Core $588 \mathrm{C}-1$ and Sample 588C-2-1, 0-1 cm. The range of Discoaster quinqueramus, Samples 588-12-2, 0-1 cm through 58820-7, 0-1 cm, defines Zone NN11. The first occurrence of Amaurolithus primus in Sample 588-18-3, 0-1 cm subdivides Zone NN11 into Subzones NN11b and NN11a. The range of $D$. hamatus, from Samples 588-23-5, 0-1 $\mathrm{cm}$ through 588-25-3, 0-1 cm, places these samples in Zone NN9 and the overlying interval, Samples 58820 , CC through 588-23-4, 0-1 cm, in Zone NN10. The successive first occurrences of Catinaster coalitus in Sample 588-25-6, 0-1 cm and D. kugleri in Sample 588A2-4, 0-1 cm place Samples 588-25-4, 0-1 cm through 588$25-6,0-1 \mathrm{~cm}$ in Zone NN8 and Samples 588-25,CC through 588A-2-4, 0-1 cm in Zone NN7. The last occur- rence of Sphenolithus heteromorphus in Sample 588A9-1, 0-1 cm places Sample 588A-2,CC and Cores 3-8 of Hole 588A in Zone NN6. The first occurrence of $D$. exilis in Sample 588C-1,CC places Samples 588A-9-1, 0-1 $\mathrm{cm}$ through $588 \mathrm{C}-1, \mathrm{CC}$ in Zone NN5. Zone NN4 includes Samples 588C-2-1, 0-1 cm through 588C-2-5, 0$1 \mathrm{~cm}$. The last occurrences of $S$. belemnos in Sample 588C-2-6, 0-1 cm and Triquetrorhabdulus carinatus in Sample 588C-3,CC place Samples 588C-2-6, 0-1 cm through 588C-3-6, 0-1 cm in Zone NN3. Zone NN2 includes Sample 588C-3,CC down to the first occurrence of D. druggii in Sample 588C-5-1, 21-22 cm. The last occurrence of Helicosphaera recta in Sample 588C-6-2, $0-1 \mathrm{~cm}$ marks the Oligocene/Miocene boundary and places Samples 588C-5-2, 0-1 cm through 588C-6-1, 0-1 $\mathrm{cm}$ in Zone NN1.

\section{Site 589}

\section{Hole 589}

Site 589 , at a water depth of $1391 \mathrm{~m}$ below sea level, is located east of the crest of Lord Howe Rise $\left(30^{\circ} 42.72^{\prime} \mathrm{S}\right.$, $163^{\circ} 38.39^{\prime} \mathrm{E}$ ) in waters transitional between subtropical and temperate water masses. One hole was drilled and four cores were obtained before the onboard positioning computer failed and drilling operations ended at a depth of $36.1 \mathrm{~m}$. Hole 589 penetrated a complete Quaternary sequence and terminated in the latest Pliocene Zone NN18 (Table 5).

Species diversity and preservation are good throughout Hole 589. However, there was considerable reworking of some species, particularly several discoaster species in Cores 3 and 4.

\section{Quaternary}

Emiliania huxleyi occurs abundantly in Samples 589$1-1,0-1 \mathrm{~cm}$ and $589-1-2,0-1 \mathrm{~cm}$. Therefore, these samples are placed in Zone NN21. The last common occurrence of E. ovata in Sample 589-1-5, 0-1 cm is taken as the top of Subzone NN19b, which places Sample 589$1-3,0-1 \mathrm{~cm}$ in Zone NN20. Rare occurrences of $E$. ovata above Sample 589-1-5, 0-1 cm are interpreted as reworking. The last consistently common occurrence of Calcidiscus macintyrei in Sample 589-3-6, 0-1 cm marks the top of Subzone NN19a, placing Samples 589-1-5, $0-1 \mathrm{~cm}$ through $589-3-5,0-1 \mathrm{~cm}$ in Subzone NN19b and Samples 589-3-6, 0-1 cm through 589-4-6, 0-1 cm in Subzone NN19a. Rare to common occurrences of $C$. macintyrei above Sample 589-3-6, 0-1 cm are interpreted as reworking.

\section{Pliocene}

The common occurrence of Discoaster brouweri in Sample 589-4,CC places this sample in the latest Pliocene Zone NN18. Rare occurrences of $D$. brouweri above this sample are interpreted as reworking.

\section{Site $\mathbf{5 9 0}$}

$$
\text { Holes 590, 590A, 590B }
$$

Site 590 is located on the crest of Lord Howe Rise $\left(31^{\circ} 10.02^{\prime} \mathrm{S}, 163^{\circ} 21.51^{\prime} \mathrm{E}\right)$ immediately north of the sub- 
Table 5. Nannoplankton occurrences, Hole 589.

\begin{tabular}{|c|c|c|c|c|c|c|c|c|c|c|c|c|c|c|c|c|c|c|c|c|c|c|c|c|c|c|c|c|c|c|c|c|c|c|c|c|}
\hline & & & & & & & & $\begin{array}{l}\text { nde } \\
\text { peci }\end{array}$ & $\begin{array}{l}\text { ex } \\
\text { ies }\end{array}$ & & & & & & & & & & & & ti & ion & al sp & ecies & & & & & & & & & & & & \\
\hline$\stackrel{\square}{4}$ & ัّ & రั & 들 & 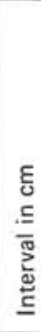 & 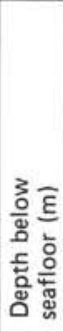 & 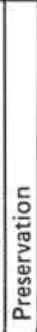 & 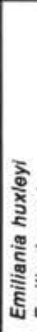 & 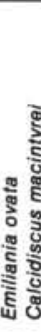 & 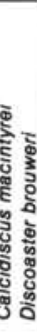 & & 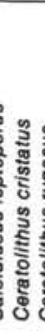 & & 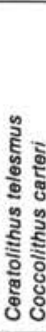 & 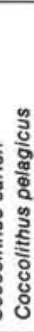 & 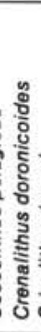 & 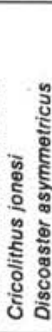 & 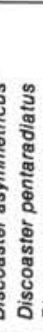 & 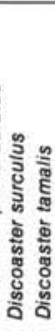 & 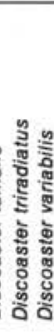 & 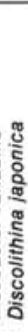 & 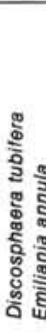 & 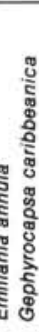 & 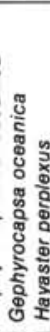 & 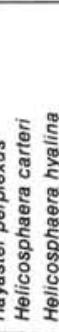 & 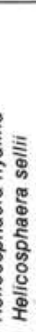 & & 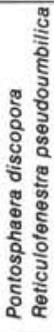 & 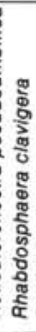 & 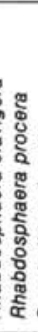 & 竧 & 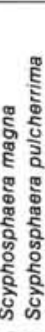 & 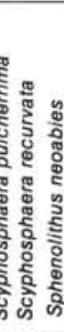 & 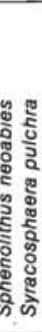 & 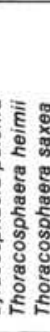 & 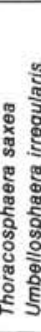 & 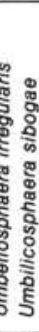 \\
\hline \multirow{24}{*}{ 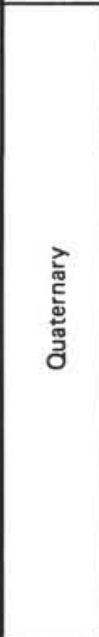 } & \multirow{2}{*}{ NN21 } & 1 & 1 & 0.1 & 001 & G & $A$ & A C & & A & & & $A R$ & A & c & & & & & $\bar{A}$ & $R$ & c & C & A C & A & & A & C & $c$ & c & & & c & A A & म म & a $C$ \\
\hline & & & 2 & 0.1 & 151 & G & A & A C & & A & A & & A R & c & c & $R$ & & & & C & $R$ & C & C & C R & A & & A A & C & c & C A & A & A & C & A C C & $c$ A & ${ }^{4} \mathrm{C}$ \\
\hline & NN20 & & 3 & $0-1$ & 301 & $G$ & & A C & & c & $A$ & & A R & A & c & A & & & & A & $R$ & C & C & C R & A & & A & R & C & $A A$ & A & A & A & R C & $C A$ & $A$ \\
\hline & \multirow{13}{*}{ NN19b } & & 5 & $0-1$ & 601 & G & & c c & & A & A & & A R & A & $A$ & A & & & & $A$ & $\bar{A}$ & $A$ & C & C R & $A$ & & A & A & $R$ & A A & A & A & R & R A & A & $\mathrm{A}$ \\
\hline & & & & $\mathrm{CC}$ & 730 & G & & c A & & A & A & & A A & A & $A$ & A & & & & $A$ & A & C & C & C R & A & & A & $A$ & $R$ & A A & A & A & $R$ & R R & A & $\mathrm{A}$ \\
\hline & & 2 & 3 & 0.1 & 1031 & G & & c $\mathrm{A}$ & & C & $A$ & RF & $R \quad R$ & $A$ & $A$ & A & & & & $A$ & A & R & A & C A & c & $R$ & C & A & $A$ & A A & A & c & A & A A & 7 $A$ & $A$ \\
\hline & & & 4 & 0.1 & 1181 & $G$ & & C $R$ & & A & R & R F & R R & A & A & R R & & & & $R$ & A & $A$ & R & C R & C & $R$ & A & $R$ & $R$ & R R & $R$ & R & A & A A & A & $A$ \\
\hline & & & 5 & 0.1 & 1331 & $G$ & & C $R$ & & C & R & $R F$ & A & R & $\mathbf{A}$ & A & & R & & $A$ & A & A & A & C R & A & A & R & A & $A$ & R A & A & R & A & A A & A $R$ & A \\
\hline & & & 6 & $0-1$ & 1481 & G & & C R & & C & A & $\overline{R F}$ & A & A & A & & & & & $A$ & A & A & A & C R & A & $R$ & A & $R$ & $R$ & R A & 8 & R & A & A A & $R$ & A \\
\hline & & & 7 & 0.1 & 1631 & G & & C R & & C & A & A & $A$ & $A$ & A & & & & & A & A & A & R & C A & $R$ & R & R & $R$ & A & $R \quad R$ & $\mathrm{R} A$ & A & A & A A & A & A \\
\hline & & & & CC & 1690 & G & & $A R$ & & C & $A$ & AF & A & $A$ & A & & $\bar{R}$ & & A & $A$ & $\bar{A}$ & $R$ & R & C R & $R$ & A & A & $R$ & $R$ & A & A & A & $A$ & A A & $A$ & $A$ \\
\hline & & 3 & 1 & 0.1 & 1691 & G & & $A R$ & R & C & A & R & & $A$ & A & & $R$ & & & $A$ & A & $R$ & $R$ & C R & $R$ & $R$ & A & $R$ & $R$ & A & & A & A & A A & A $\mathrm{A}$ & $A$ \\
\hline & & & 2 & 0.1 & 1841 & $G$ & & $A \quad R$ & a $\mathrm{A}$ & C & A & A & A & A & A & R & & & & $A$ & C & R & $R$ & C A & A & A & $A$ & $R$ & $A$ & A & & R & A & A A & $A$ & $A$ \\
\hline & & & 3 & $0-1$ & 1991 & G & & $A$ A & $R$ & C & A & A F & A R & A & A & & & & R & A & C & R & A & C R & A & A & R & R & A & R & A & A & R & A $A$ & A $R$ & A \\
\hline & & & 4 & 0.1 & 2141 & G & & $A$ A & $R$ & A & A & A F & A & A & A & & R & & & A & C & A & A & C & A & $R$ & A & R & A & R & & R & A & A P & A $\mathrm{A}$ & A \\
\hline & & & 5 & $0-1$ & 2291 & G & & C A & A & A & A & $R$ & & A & A & & $A$ & & & $A$ & C & A & A & C & $A$ & $R$ & A & $R$ & $R$ & A & & A & A & A $A$ & A & A \\
\hline & \multirow{8}{*}{ NN19a } & & 6 & 0.1 & 2441 & 6 & & $A C$ & $C$ A & A & A & A & A & A & A & & A & & & A & C & A & R & c & A & $R$ & R & A & A & A & & A & A & A $A$ & A $R$ & $A$ \\
\hline & & & & CC & 2617 & G & & A C & C $\mathrm{A}$ & A & $R$ & RF & A & A & A & A & $A$ & & & A & C & A & A & C & A & $A$ & A & A & A & A & & R & A & A R & A $\mathrm{A}$ & $A$ \\
\hline & & 4 & 1 & 0.1 & 2651 & G & & $A C$ & C & A & A & A & A & A & A & & R & A & & A & C & c & C & c & c & A & R & A & A & A & & R R & A & A A & A $\mathrm{A}$ & $A$ \\
\hline & & & 2 & 0.1 & 2801 & $G$ & & A C & EA & A & $A$ & R F & R R & A & A & & $A$ & A & & A & A & $\mathrm{C}$ & $A R$ & C & C & $A$ & R & R & A & C & $A$ & R & C & A & A & \\
\hline & & & 3 & 0.1 & 2951 & $G$ & & A C & $C R$ & A & A & R F & A $R$ & A & A & & 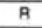 & A A & $A$ & $A$ & A & A & & c & R & A & & A & A & R & & $R$ & A & A & A & \\
\hline & & & 4 & 0.1 & 3101 & $G$ & & A C & $C R$ & A & A & A F & R R & C & A & & $A$ & A & & A & C & A & & C & C & R & & A & A & A & & R & A & A & A & \\
\hline & & & 5 & 0.1 & 3251 & 6 & & c c & $\bar{C}$ & A & A & A & $R$ R & C & A & & $A$ & $A$ & A R & A & $\bar{A}$ & & & C & c & A & & A & R & R & & R & $A$ & A & A & \\
\hline & & & \begin{tabular}{|l|}
6 \\
\end{tabular} & 0. & 3401 & G & & A C & C $R$ & A & A & & A & C & A & & $A$ & A & A & A & C & & & C & C & $A$ & & $A$ & A & A & & R & C & A & A & \\
\hline io. late & NN1B & & & $\mathrm{CC}$ & 3610 & $G$ & & $A C$ & c c & & $R$ & A & R A & C & A & & $R$ & A & C R & A & C & & & C & C & R & & $R$ & A & A & A & & c & A & R & \\
\hline
\end{tabular}

tropical divergence at a water depth of $1299 \mathrm{~m}$ below sea level. Site 590 consists of three holes: Hole 590 was cored to a depth of $26.2 \mathrm{~m}$ and three cores were taken; Hole 590A was washed down to the depth of Hole 590, then cored to a depth of $280.8 \mathrm{~m}$, and 27 cores were obtained; and Hole 590B was cored to a depth of $499.1 \mathrm{~m}$ and 53 cores were taken. Core recovery was good throughout the three holes.

In order to obtain a complete stratigraphic section, the three cores from Hole 590 together with 18 cores from Hole 590A and 31 cores from Hole 590B were examined for calcareous nannoplankton. All of the Neogene and Quaternary nannoplankton zones are present at Site 590 (Table 6). Species diversity is good throughout Site 590 and little reworking was encountered. Preservation is good down through Sample 590A-14-1, 0-1 $\mathrm{cm}$ and fair below this level.

\section{Quaternary}

The Quaternary includes Cores 1-3 of Hole 590 and Core 1 of Hole 590A. The presence of Emiliania huxleyi in Samples 590-1-1, 5-6 cm through 590-1-3, 5-6 cm places these samples in Zone NN21. The last occurrence of E. ovata in Sample 590-1,CC places Samples 590-1-4, 5-6 cm and 590-1-5, 5-6 cm in Zone NN20. The last occurrence of Discoaster brouweri in Sample 590A-2-1, 5$6 \mathrm{~cm}$ places Samples 590-1,CC through 590A-1, CC in Zone NN19. The last occurrence of Calcidiscus macintyrei in Sample 590-3-3, 5-6 cm subdivides Zone NN19 into Subzones NN19b and NN19a. A rare occurrence of C. macintyrei is reworked in Sample 590-1-1, 5-6 cm.
Rare occurrences of several discoaster species are reworked into Subzone NN19a and Zone NN18.

\section{Pliocene}

The interval from Core 2 of Hole 590A through Sample 590A-18-5, 5-6 cm belongs in the Pliocene. The lower/upper Pliocene boundary is placed between Samples $590 \mathrm{~A}-7-3,0-1 \mathrm{~cm}$ and $590 \mathrm{~A}-7-4,0-1 \mathrm{~cm}$. The last consistent occurrences of Discoaster brouweri in Sample 590A-2-1, 5-6 cm, D. pentaradiatus in Sample 590A3-1, 5-6 cm, D. surculus in Sample 590A-3-2, 5-6 cm, and Reticulofenestra pseudoumbilica in Sample 590A$7-4,0-1 \mathrm{~cm}$ place Samples 590A-2-1, 5-6 cm through $590 \mathrm{~A}-2, \mathrm{CC}$ in Zone NN18, Sample 590A-3-1, 5-6 cm in Zone NN17, and Samples 590A-3-2, 5-6 cm through 590A-7-3, 0-1 cm in Zone NN16. The interval between the last occurrence of $R$. pseudoumbilica in Sample 590A-7-4, 0-1 cm and the last occurrence of Amaurolithus tricorniculatus in Sample 590A-11-7, 0-1 cm belongs in Zone NN15. Zone NN14 includes Samples 590A$11, \mathrm{CC}$ through $590 \mathrm{~A}-14-1,0-1 \mathrm{~cm}$, the first occurrence of D. asymmetricus. Ceratolithus rugosus has its first occurrence in Sample 590A-16-2, 5-6 cm, thus placing Samples 590A-14-2, 0-1 cm through 590A-16-2, 5-6 cm in Zone NN13. Zone NN12 includes samples from 590A$16-3,5-6 \mathrm{~cm}$ down to Sample 590A-18-5, 5-6 cm.

\section{Miocene}

The Miocene includes the interval from Sample 590A$18-6,5-6 \mathrm{~cm}$ through 590B-53,CC. The middle/upper Miocene boundary lies between Sample 590B-38-5, 5- 
Table 6. Nannoplankton occurrences, Holes 589, 590A, and 590B.

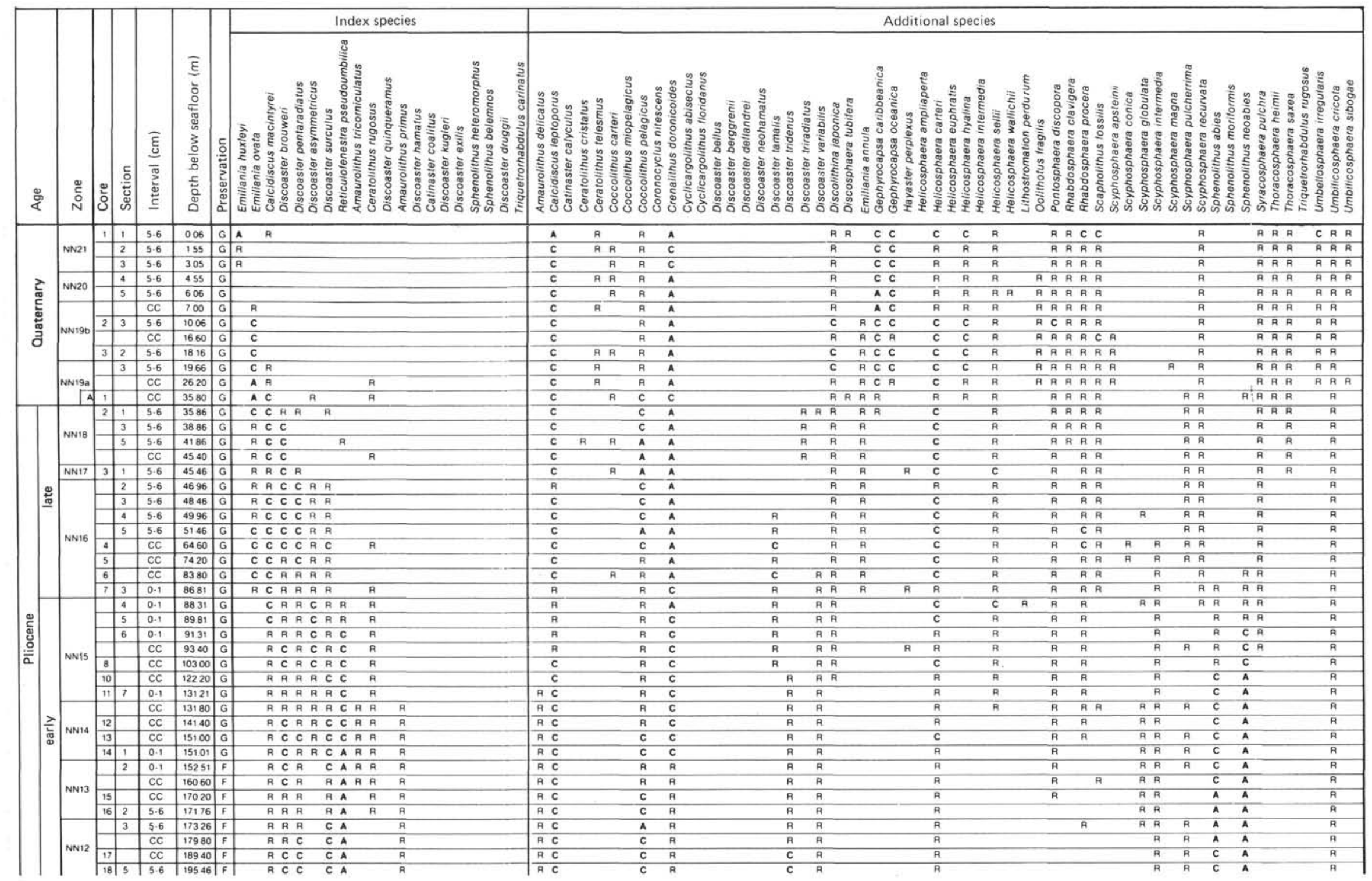




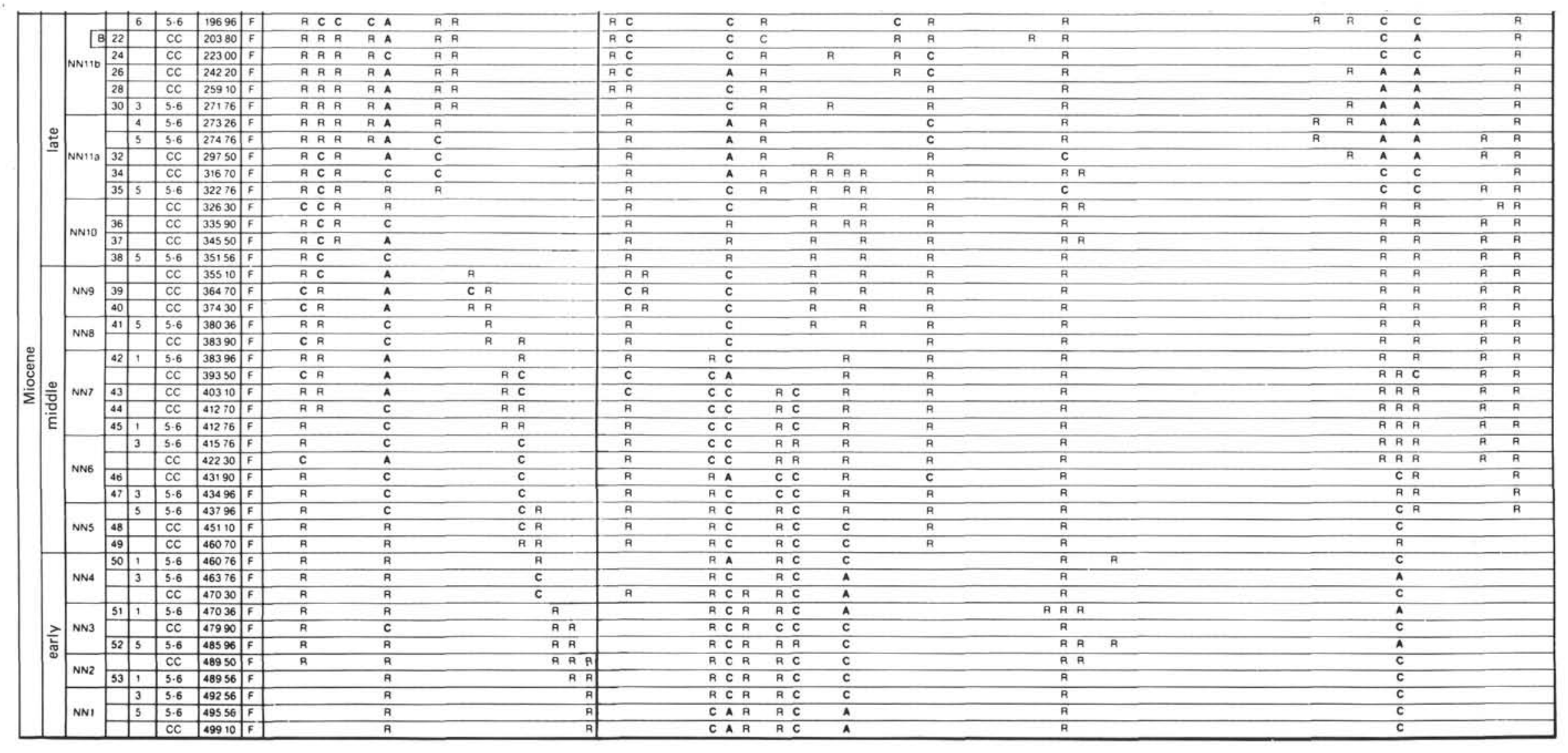


$6 \mathrm{~cm}$ and 590B-38,CC and the lower/middle Miocene boundary is placed between Samples 590B-49, CC and 590B-50-1, 5-6 cm. The range of Discoaster quinqueramus from Samples 590A-18-6, 5-6 cm through 590B$35-5,5-6 \mathrm{~cm}$ defines Zone NN11. The first occurrence of Amaurolithus primus in Sample 590B-30-3, 5-6 cm subdivides this zone into Subzones NN11b and NN11a. The range of $D$. hamatus from Samples 590B-38,CC through 590B-40, CC places these samples in Zone NN9 and Samples 590B-35, CC through 590B-38-5, 5-6 cm in Zone NN10. The first occurrences of Catinaster coalitus in Sample 590B-41,CC and D. kugleri in Sample 590B45-1, 5-6 cm place Samples 590B-41-5, 5-6 cm and 590B-41, CC in Zone NN8 and Samples 590B-42-1, 5-6 $\mathrm{cm}$ through $590 \mathrm{~B}-45-1,5-6 \mathrm{~cm}$ in Zone NN7. The interval from Sample 590B-45-3, 5-6 cm to Sample 590B47-3, 5-6 cm, above the last occurrence of Sphenolithus heteromorphus in Sample 590B-47-5, 5-6 cm, belongs in Zone NN6. The interval from Sample 590B-47-5, 5-6 $\mathrm{cm}$ to 590B-49,CC, the first occurrence of $D$. exilis, is placed in Zone NN5. Although Helicosphaera ampliaperta is normally absent in samples from the western Pacific, a single specimen was observed in Sample 590B$51-1,5-6 \mathrm{~cm}$. The last occurrence of $S$. belemnos in Sample 590B-51-1, 5-6 cm places Core 50 in Zone NN4. The last occurrence of Triquetrorhabdulus carinatus in Sample 590B-52, CC and the first occurrence of Discoaster druggii in Sample 590B-53-1, 5-6 cm place Samples 590B-51-1, 5-6 cm through 590B-52-5, 5-6 cm in Zone NN3, Samples 590B-52,CC and 590B-53-1, 5-6 $\mathrm{cm}$ in Zone NN2, and Sample 590B-53-3, 5-6 cm through 590B-53,CC in Zone NN1.

\section{Site 591}

\section{Holes 591, 591B}

Site 591 is located on a spur of Lord Howe Rise $\left(31^{\circ} 35.06^{\prime} \mathrm{S}, 164^{\circ} 26.92^{\prime} \mathrm{E}\right)$ and lies on the subtropical divergence at a water depth of $2131 \mathrm{~m}$ below sea level. Three holes were drilled at Site 591. Hole 591 was cored to a depth of $283.1 \mathrm{~m}$ and 31 cores were taken. Hole $591 \mathrm{~A}$, a duplication of Hole 591, was cored to a depth of $284.6 \mathrm{~m}$ and 30 cores were taken. Hole 591B was washed down to a depth of $270.6 \mathrm{~m}$ and then cored to a depth of $500.4 \mathrm{~m}$. Twenty-four cores were taken. Core recovery was good throughout Hole 591 and most of Hole 591A and fair throughout Hole 591B.

In order to obtain a complete stratigraphic section, 31 cores from Hole 591 and 22 cores from Hole 591B were examined for calcareous nannoplankton. Species diversity is good throughout Site $\mathbf{5 9 1}$ and reworking is minimal. Preservation of calcareous nannoplankton is good throughout Cores 1-16 of Hole 591 and fair below this level. Hole 591B terminated in the early Miocene Zone NN4 and all of the overlying Neogene and Quaternary nannoplankton zones are recognized (Table 7).

\section{Quaternary}

The Quaternary includes Samples 591-1-1, 4-5 cm through 591-6-5, 3-4 cm. The occurrence of Emiliania huxleyi in Samples 591-1-1, 4-5 cm and 591-1-3, 4-5 cm places these samples in Zone NN21. The last common occurrence of $E$. ovata in Sample 591-2-5, 3-4 cm marks the top of Subzone NN19b. A rare occurrence of $E$. ovata above this level is interpreted as reworking. The intervening samples belong in Zone NN20. The last consistent occurrence of Calcidiscus macintyrei in Sample 591-5-5, 3-4 cm marks the top of Subzone NN19a. A single occurrence of C. macintyrei in Sample 591-1,CC is interpreted as reworking.

\section{Pliocene}

The Pliocene includes Samples 591-6,CC through 59124-1, 3-4 cm. The lower/upper Pliocene boundary is located between Samples 591-12-3, 3-4 cm and 591-12-5, $3-4 \mathrm{~cm}$. The top of Zone NN18 is marked by the last occurrence of Discoaster brouweri in Sample 591-6,CC. Zone NN18 extends down to the last occurrence of $D$. pentaradiatus in Sample 591-8-3, 3-4 cm. This sample is placed in Zone NN17. The last occurrence of $D$. surculus in Sample 591-8-5, 3-4 cm down to the last consistent occurrence of Reticulofenestra pseudoumbilica in Sample 591-12-5, 3-4 cm places Sample 591-8-5, 3-4 cm through $591-12-3,3-4 \mathrm{~cm}$ in Zone NN16. Two occurrences of $R$. pseudoumbilica above this level are interpreted as reworking. The interval from the last occurrence of Amaurolithus tricorniculatus in Sample 59117-1, 21-22 cm through Sample 591-20,CC, the first occurrence of Discoaster asymmetricus, is placed in Zone NN14. The next two samples, down to the first occurrence of Ceratolithus rugosus in Sample 591-21-5, 3$4 \mathrm{~cm}$, belong in Zone NN13. The interval from Sample 591-21,CC to Sample 591-24-1, 3-4 cm, above the last occurrence of $D$. quinqueramus in Sample 591-24-3, 3-4 $\mathrm{cm}$, is placed in Zone NN12.

\section{Miocene}

The Miocene ranges from Sample 591-24-3, 3-4 cm through Sample 591B-24,CC. Hole 591B penetrated just into the early Miocene Zone NN4. The middle/upper Miocene boundary lies between Samples 591B-8,CC and 591B-9-2, 4-5 cm and the lower/middle Miocene boundary lies between Samples 591B-23,CC and 591B-24-1, 3-4 cm. The range of Discoaster quinqueramus, down through Sample 591B-4,CC, defines Zone NN11. The first consistent occurrence of Amaurolithus primus in Sample 591-28-3, 3-4 cm marks the boundary between Subzones NN11b and NN11a. The interval from Sample 591B-5-1, 3-4 cm through Sample 591B-8,CC is placed in Zone NN10. The range of $D$. hamatus, from Sample 591B-9-2, 4-5 cm through Sample 591B-11-1, 4-5 cm, places these samples in Zone NN9. The occurrence of Catinaster coalitus in Sample 591B-11,CC places this sample in Zone NN8. The next two samples belong in Zone NN7, based on the first occurrence of D. kugleri in Sample 591B-12,CC. Zone NN6 includes Samples 591B$13-1,3-4 \mathrm{~cm}$ through 591B-18-3, 3-4 cm. The interval from the last occurrence of Sphenolithus heteromorphus in Sample 591B-18-5, 3-4 cm down to the first occurrence of $D$. exilis in Sample 591B-23,CC is placed in Zone NN5. Core 24 belongs in Zone NN4. 
Table 7. Nannoplankton occurrences, Holes 591 and 591B.

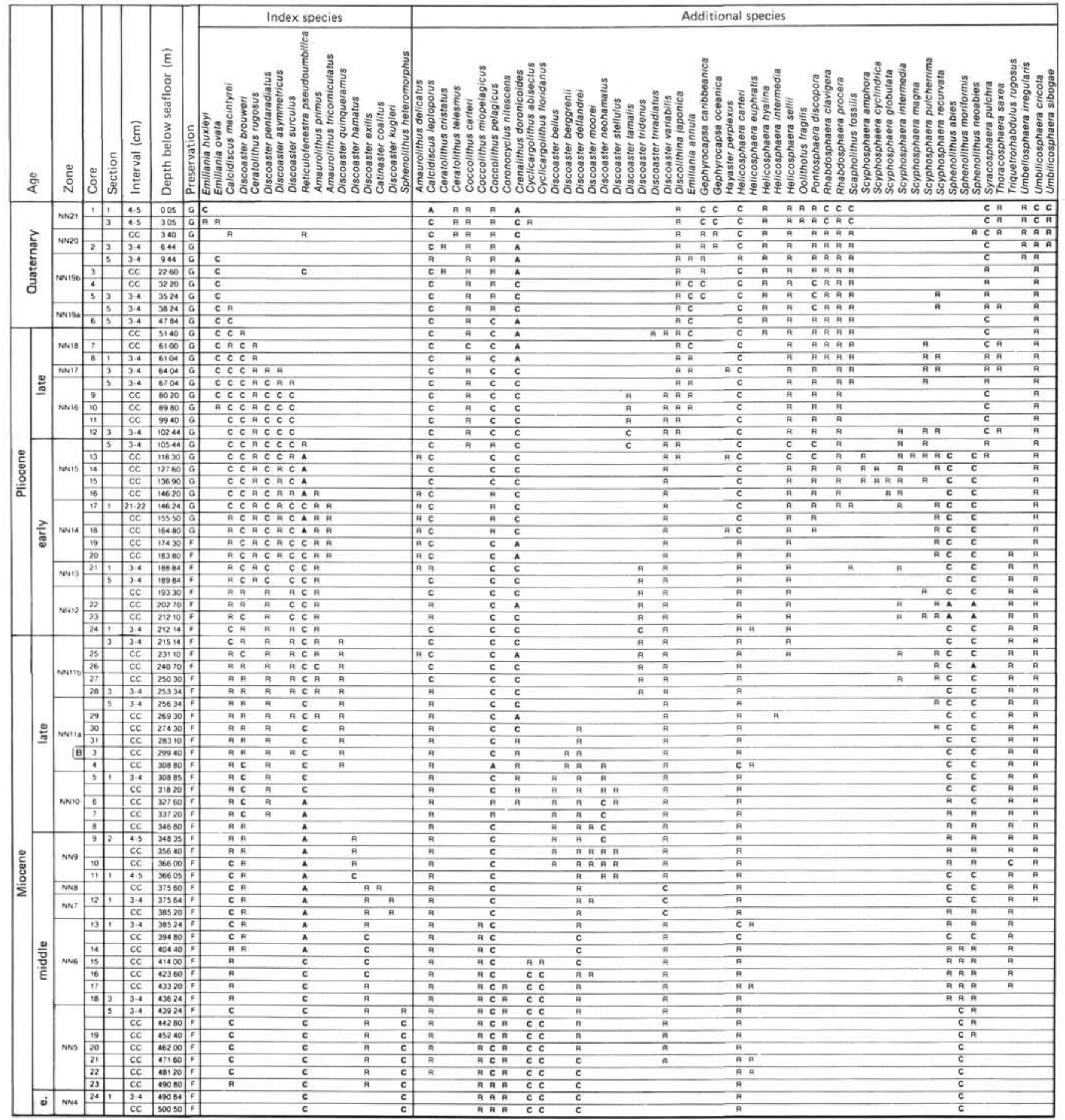

\section{Site $\mathbf{5 9 2}$}

\section{Hole 592}

Site 592 is located on the southern Lord Howe Rise $\left(36^{\circ} 28.40^{\prime} \mathrm{S}, 165^{\circ} 26.53^{\prime} \mathrm{E}\right)$ at a water depth of $1098 \mathrm{~m}$ below sea level. Hole 592, which lies in the temperate water mass, was cored to a depth of $388.5 \mathrm{~m}$ (late Eocene Zone NP18). Forty-one cores were taken and recovery was good throughout the hole.
The major regional unconformity of the southwest Pacific was encountered within Core 33 at a depth of $306 \mathrm{~m}$. It separates lower Oligocene sediment (Zone NP22) from immediately overlying lower Miocene sediment (Zone NN2). Except for the boundary between the early Pliocene Zones NN13 and NN12, the Neogene and Quaternary nannoplankton zonation is complete down to the unconformity (Table 8). The preservation of calcareous nannoplankton in Hole 592 is good in Cores 1-4 
Table 8. Nannoplankton occurrences, Hole 592.

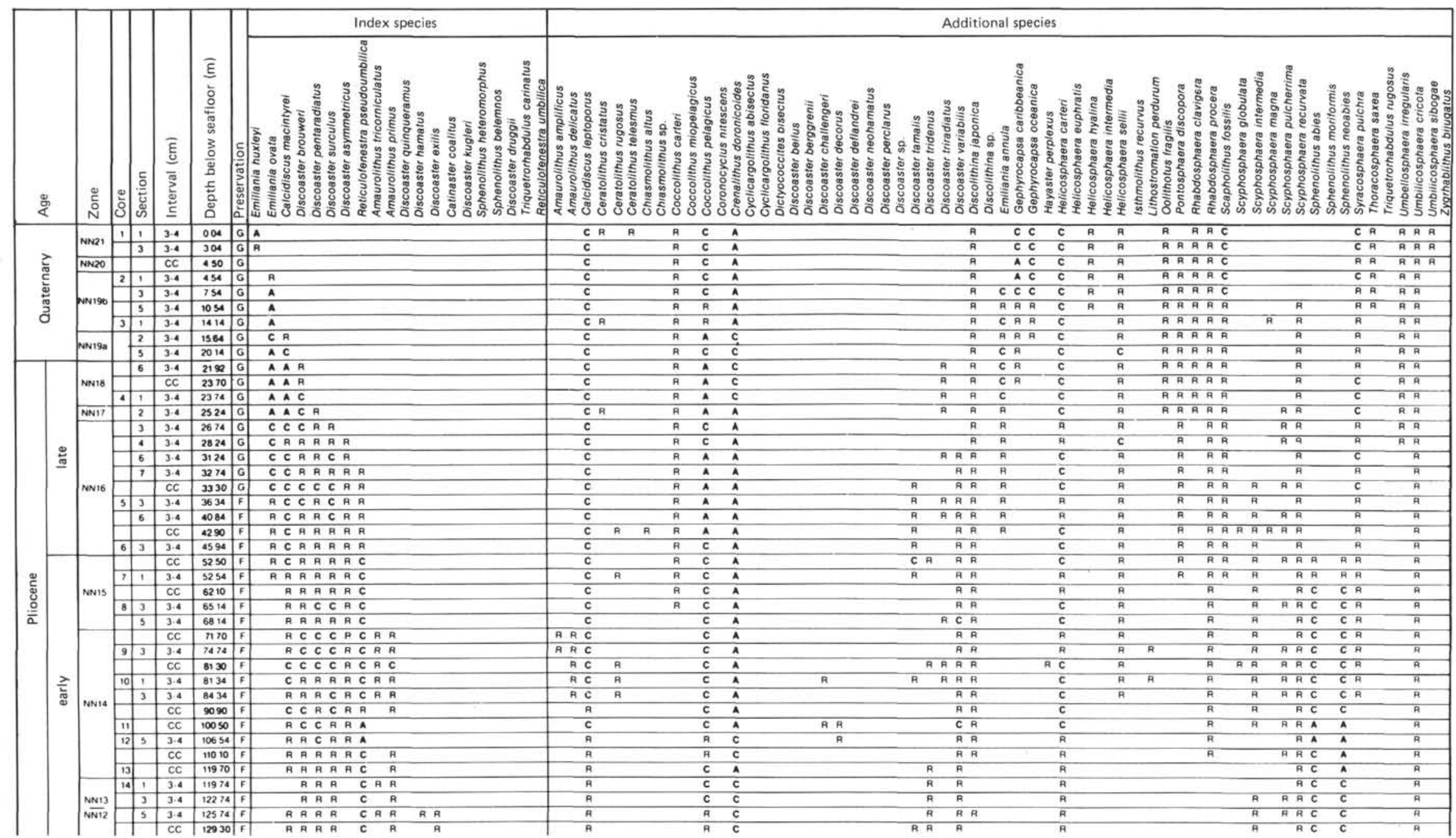




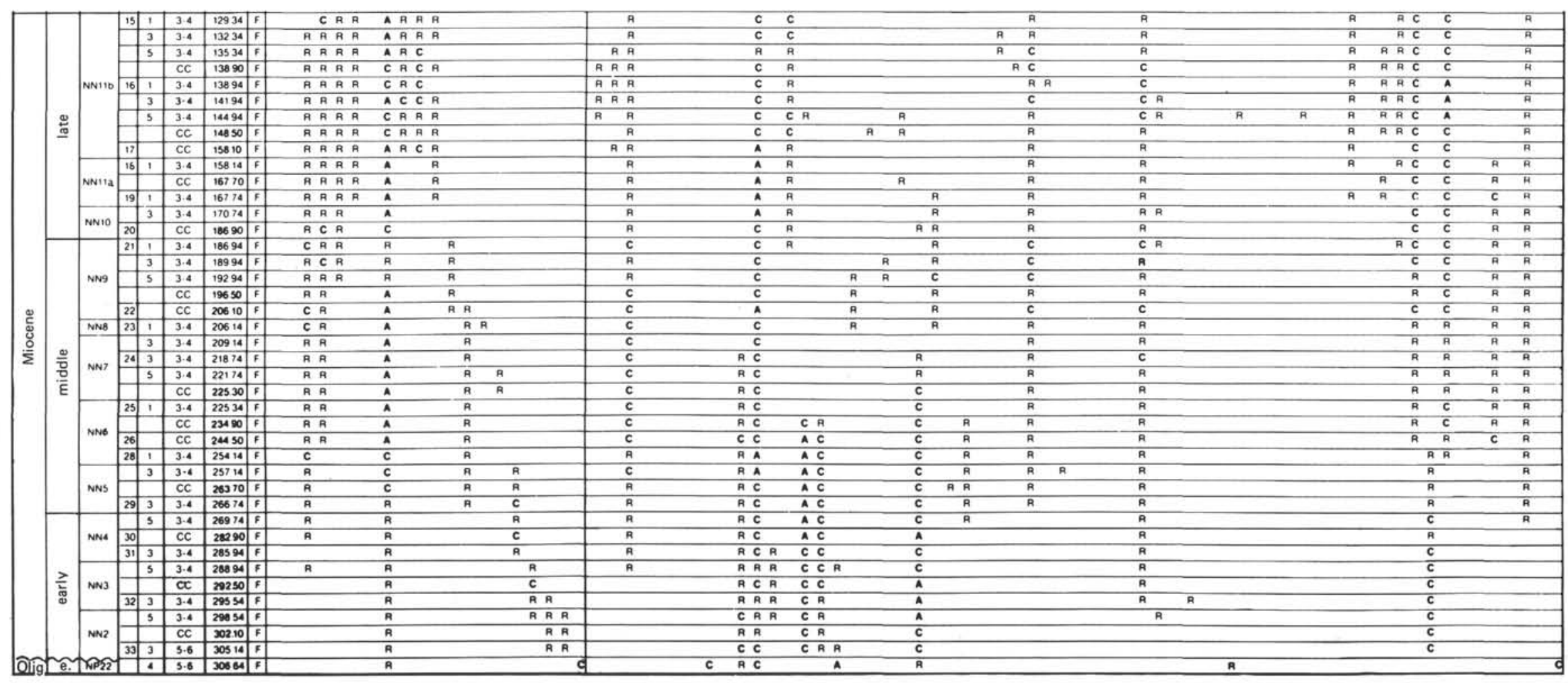


and fair in Cores 5-33. Species diversity is good throughout these cores.

\section{Quaternary}

The Quaternary includes Samples 592-1-1, 3-4 cm through 592-3-5, 3-4 cm. Zone NN21, based on the range of Emiliania huxleyi, includes Samples 592-1-1, 3-4 $\mathrm{cm}$ and 592-1-3, 3-4 cm. Sample 592-1,CC, above the last occurrence of E. ovata in Sample 592-2-1, 3-4 $\mathrm{cm}$, belongs in Zone NN20. The last occurrence of Calcidiscus macintyrei in Sample 592-3-2, 3-4 cm places Samples 592-2-1, 3-4 cm to 592-3-1, 3-4 cm in Subzone NN19b. Subzone NN19a includes Samples 592-3-2, 3-4 $\mathrm{cm}$ and 592-3-5, 3-4 cm, above the last occurrence of Discoaster brouweri in Sample 592-3-6, 3-4 cm.

\section{Pliocene}

The Pliocene includes Sample 592-3-6, 3-4 cm through Core 14. The lower/upper Pliocene boundary is between Samples 592-6-3, 3-4 cm and 592-6,CC. The last occurrence of Discoaster pentaradiatus in Sample 592-4-2, 3$4 \mathrm{~cm}$ places Samples 592-3-6, 3-4 cm to 592-4-1, 3-4 cm in Zone NN18. One sample, Sample 592-4-2, 3-4 cm, belongs in Zone NN17. The interval between the last occurrence of $D$. surculus in Sample 592-4-3, 3-4 cm and the last common occurrence of Reticulofenestra pseudoumbilica in Sample 592-6,CC is placed in Zone NN16. Rare occurrences of $R$. pseudoumbilica above Sample $592-6, C C$ are interpreted as reworking. The last occurrence of Amaurolithus tricorniculatus in Sample 592$8, \mathrm{CC}$ places Samples $592-6, \mathrm{CC}$ to $592-8-5,3-4 \mathrm{~cm}$ in Zone NN15. Samples 592-8,CC through 592-13,CC, the first occurrence of $D$. asymmetricus, are placed in Zone NN14. The paucity of Ceratolithus rugosus at Site 592 prevents determination of the NN13/NN12 boundary.

\section{Miocene}

Samples 592-15-1, 3-4 cm through 592-33-3, 5-6 cm are in the Miocene. The middle/upper Miocene boundary is between Samples 592-20,CC and 592-21-1, 3-4 cm and the lower/middle Miocene boundary is between Samples 592-29-3, 3-4 cm and 592-29-5, 3-4 cm. The range of Discoaster quinqueramus from Sample 592-15-1, 3-4 $\mathrm{cm}$ to Sample 592-19-1, 3-4 cm determines Zone NN11. The first occurrence of Amaurolithus primus in Sample 592-17, CC subdivides Zone NN11 into Subzones NN11b and NN11a. The range of $D$. hamatus from Sample 592-21-1, 3-4 cm to Sample 592-22,CC places this inter$\mathrm{val}$ in Zone NN9. The overlying interval belongs in Zone NN10. The first occurrence of Catinaster coalitus in Sample 592-23-1, 3-4 cm places this sample in Zone NN8. Zone NN7 includes Sample 592-23-3, 3-4 cm to the first occurrence of D. kugleri in Sample 592-24,CC. Sphenolithus heteromorphus last occurs in Sample 592-28-3, 3$4 \mathrm{~cm}$, placing Samples 592-25-1, 3-4 cm through 592$28-1,3-4 \mathrm{~cm}$ in Zone NN6. The interval from Sample $592-28-3,3-4 \mathrm{~cm}$ to the first occurrence of $D$. exilis in Sample 592-29-3, 3-4 cm is placed in Zone NN5. Samples $592-29-5,3-4 \mathrm{~cm}$ to $592-31-3,3-4 \mathrm{~cm}$, above the last occurrence of $S$. belemnos in Sample 592-31-5, 3-4 $\mathrm{cm}$, belong in Zone NN4. The last occurrence of Triquetrorhabdulus carinatus in Sample 592-32-5, 3-4 cm plac- es Samples 592-31-5, 3-4 cm through 592-32-3, 3-4 cm in Zone NN3. Based upon the occurrence of $D$. druggii in Sample 592-33-3, 5-6 cm and the common occurrence of Reticulofenestra umbilica in Sample 592-33-4, $5-6 \mathrm{~cm}$, the major regional unconformity of the southwest Pacific occurs between these samples, with early Miocene Zone NN2 lying immediately above early Oligocene Zone NP22. The missing NN1 and NP25/NP23 zones represent a hiatus of at least 15.5 m.y.

\section{Site $\mathbf{5 9 3}$}

\section{Hole 593}

Site 593 is located on the Challenger Plateau west of the northern tip of the South Island of New Zealand $\left(40^{\circ} 30.47^{\prime} \mathrm{S}, 167^{\circ} 40.47^{\prime} \mathrm{E}\right)$ at a water depth of $1068 \mathrm{~m}$ below sea level. Site 593, which lies in the temperate water mass, is a reoccupation of Site 284 (DSDP Leg 29).

Two holes were drilled at Site 593. Hole 593 was cored to a depth of $571.5 \mathrm{~m}$ (late Eocene Zone NP18) and 60 cores were taken. Core recovery was good throughout most intervals. Hole 593A was cored to a depth of 209.3 $\mathrm{m}$; then washed down to a depth of $448.8 \mathrm{~m}$, where coring was resumed to a depth of $496.9 \mathrm{~m}$. Twenty-seven cores were taken.

Only Cores 1-50 from Hole 593 were examined in order to determine the Neogene and Quaternary nannoplankton zonation, because the Oligocene/Miocene boundary occurs within Core 50 (Table 9). Preservation of calcareous nannoplankton in Hole 593 is good in Cores 1-11 and fair in Cores 12-50. Species diversity is good throughout the hole. With the exception of the NN13/NN12 and the NN8/NN7 boundaries, all of the Neogene and Quaternary nannoplankton zones are recognized. Hole 284 (DSDP Leg 29) was cored to a depth of $208.0 \mathrm{~m}$ (late Miocene). The placement of the lower/ upper Pliocene and the upper Pliocene/Quaternary boundaries in Hole 593 and in Hole 284, as determined by Edwards and Perch-Nielsen (1973), agrees very closely. The Miocene/Pliocene boundary in Hole 284 was not recognized. Species diversity in upper Miocene/Quaternary sediments in Hole 593 is somewhat greater than in Hole 284.

\section{Quaternary}

The Quaternary includes Sample 593-1-1, 3-4 cm through Core 5. Samples 593-1-1, 3-4 cm and 593-1-3, 3-4 cm contain common to abundant Emiliania huxleyi and are placed in Zone NN21. The last occurrence of $E$. ovata in Sample 593-2-5, 3-4 cm places Samples 593-1,CC through 593-2-3, 3-4 cm in Zone NN20. Subzone NN19b includes Samples 593-2-5, 3-4 cm through 593-3-1, 3-4 $\mathrm{cm}$, above the last occurrence of Calcidiscus macintyrei in Sample 593-3-3, 3-4 cm. Subzone NN19a extends down through Core 5 .

\section{Pliocene}

The Pliocene includes Samples 593-6-1, 39-40 cm through 593-17-3, 3-4 cm. The lower/upper Pliocene boundary lies between Samples 593-8,CC and 593-9-1, 3-4 cm. The last occurrence of Discoaster brouweri in Sample 593-6-1, 39-40 cm marks the top of Zone NN18. 
The two samples below contain the last occurrences of $D$. pentaradiatus and $D$. surculus, respectively, placing Sample 593-6-2, 3-4 cm in Zone NN17. The last occurrence of Reticulofenestra pseudoumbilica in Sample 5939-1, 3-4 cm places Samples 593-6-3, 3-4 cm through $593-8, C C$ in Zone NN16. Zone NN15 includes all of Core 9. The last occurrence of Amaurolithus tricorniculatus in Sample 593-11-1, 3-4 cm and the first occurrence of $D$. asymmetricus in Sample 593-13,CC place Cores 11-13 in Zone NN14. The NN13/NN12 boundary cannot be recognized because Ceratolithus rugosus is absent at Site 593 .

\section{Miocene}

The Miocene includes Samples 593-17-5, 3-4 cm through 593-50-3, 3-4 cm. The middle/upper Miocene boundary is between Samples 593-26-5, 3-4 cm and 59326, CC and the lower/middle Miocene boundary lies between Samples 593-43,CC and 593-44-1, 3-4 cm. The last occurrence of Discoaster quinqueramus in Sample 593-17-5, 3-4 cm and the first occurrence of Amaurolithus primus in Sample 593-22-3, 3-4 cm places this interval in Subzone NN11b. Subzone NN11a extends from Sample 593-22,CC down to 593-25-1, 3-4 cm, the first occurrence of $D$. quinqueramus. Samples 593-26,CC and 593-27, CC contain $D$. hamatus, which places this inter$\mathrm{val}$ in Zone NN9. The intervening Samples 593-25-3, 3$4 \mathrm{~cm}$ through 593-26-5, 3-4 cm belong in Zone NN10. The absence of Catinaster coalitus at Site 593 prevents the determination of the NN8/NN7 boundary. The first occurrence of D. kugleri in Sample 593-37-1, 3-4 cm and the last occurrence of Sphenolithus heteromorphus in Sample 593-42-4, 99-100 cm place Samples 593-37-3, 3-4 cm through 593-42-4, 3-4 cm in Zone NN6. The interval from Sample 593-42-4, 99-100 cm through Sample 593-43, CC, the first occurrence of $D$. exilis, belongs in Zone NN5. The last occurrence of $S$. belemnos in Sample 593-44,CC places Samples 593-44-1, 3-4 cm and 593-44-5, 3-4 cm in Zone NN4. Zone NN3 includes samples above the last occurrence of Triquetrorhabdulus carinatus and the first occurrence of $D$. druggii in Sample 593-46,CC, which here constitutes Zone NN2. Zone NN1 extends down to the Oligocene/Miocene boundary between Samples 593-50-3, 3-4 cm and 593$50-5,3-4 \mathrm{~cm}$. The top of Zone NP25 is based on the last occurrence of Helicosphaera recta in Sample 593-50-5, $3-4 \mathrm{~cm}$.

\section{Site 594}

\section{Holes 594, 594A}

Site 594 is located on the southern margin of Chatham Rise east of the South Island of New Zealand $\left(45^{\circ} 31.41^{\prime} \mathrm{S}, 174^{\circ} 56.88^{\prime} \mathrm{E}\right)$ at a water depth of $1204 \mathrm{~m}$ below sea level. The site lies in the subantarctic water mass south of the Subtropical Convergence and is the southernmost site of Leg 90.

Three holes were drilled at Site 594. Hole 594 reached the middle Miocene NN5/NN6 zones at a depth of 505.1 $\mathrm{m}$. Fifty-three cores were taken and core recovery through Core $31(293.9 \mathrm{~m})$ was generally good; however, below Core 31 , core recovery was poor. Twenty-six cores were obtained from Hole 594A which duplicates portions of Hole 594 and extends the total depth down to $639.5 \mathrm{~m}$ (Zone NN5). Core recovery below $495.5 \mathrm{~m}$ (top of Core 12) was generally poor. Hole 594B was drilled to a depth of $42.9 \mathrm{~m}$ and 5 cores were obtained.

Cores 1-53 from Hole 594 and Cores 13-26 from Hole $594 \mathrm{~A}$ were examined in order to determine the nannoplankton biostratigraphy at Site 594 . Because Site 594 is near the South Island of New Zealand, numerous Paleogene as well as some Neogene taxa are reworked into indigenous assemblages. Preservation is fair throughout the site but discoasters are poorly preserved and their numbers are markedly reduced. Most of the nannoplankton zones are recognized; however, because of the paucity of zonal indicators, the Miocene and Pliocene zonal boundaries are shown with dashed lines (Table 10). Zones NN18, NN17, NN13, and the boundary between NN8 and NN7 cannot be determined.

\section{Quaternary}

The Quaternary includes Samples 594-1-1, 3-4 cm through at least 594-11-5, 3-4 cm. The base of the Quaternary cannot be determined. Samples 594-1-1, 3-4 cm through 594-6-1, 25-26 cm contain Emiliania huxleyi and are assigned to Zone NN21. The last occurrence of E. ovata in Sample 594-7,CC places Samples 594-6-3, 3-4 cm through 594-7-1, 3-4 cm in Zone NN20. Subzone NN19b includes Samples 594-7,CC through 594$11-3,3-4 \mathrm{~cm}$, above the last occurrence of Calcidiscus macintyrei in Sample 594-11-5, 3-4 cm.

\section{Pliocene}

The top of the Pliocene cannot be determined. The lower/upper Pliocene boundary lies between Samples 594-18-1, 3-4 cm and 594-18-3, 3-4 cm and the base of the Pliocene is between Samples 594-23-5, 3-4 cm and 594-23,CC. The last common occurrence of Reticulofenestra pseudoumbilica in Sample 594-18-3, 3-4 cm marks the top of Zone NN15. The presence of $R$. pseudoumbilica above this sample is interpreted as reworking. The intervening zones and subzones (NN19a-NN16) cannot be determined. A single rare occurrence of Amaurolithus tricorniculatus in Sample 594-20,CC places Samples 594-18-3, 3-4 cm through 594-20-5, 3-4 cm in Zone NN15. The boundaries between Zones NN14 and Zones NN13 and between Zones NN13 and NN12 cannot be recognized.

\section{Miocene}

The Miocene includes Samples 594-23,CC through 594A-26,CC. The middle/upper Miocene boundary lies between Samples 594-28-3, 3-4 cm and 594-20-5, 3-4 $\mathrm{cm}$. The rare occurrences of Discoaster quinqueramus in Samples 594-23,CC and 594-26-5, 3-4 cm place this interval in Zone NN11, but the absence of Amaurolithus primus at Site 594 prevents the subdivision of this zone. The rare occurrences of $D$. hamatus in Samples 594-28-5, 3-4 cm and 594-29, CC place this interval in Zone NN9. Samples 594-26,CC through 594-28-3, 3-4 $\mathrm{cm}$ therefore belong in Zone NN10. The boundary between Zones NN8 and NN7 cannot be determined. The rare occurrences of $D$. kugleri in Sample 594-42, 3-4 cm 
Table 9. Nannoplankton occurrences, Hole 593.

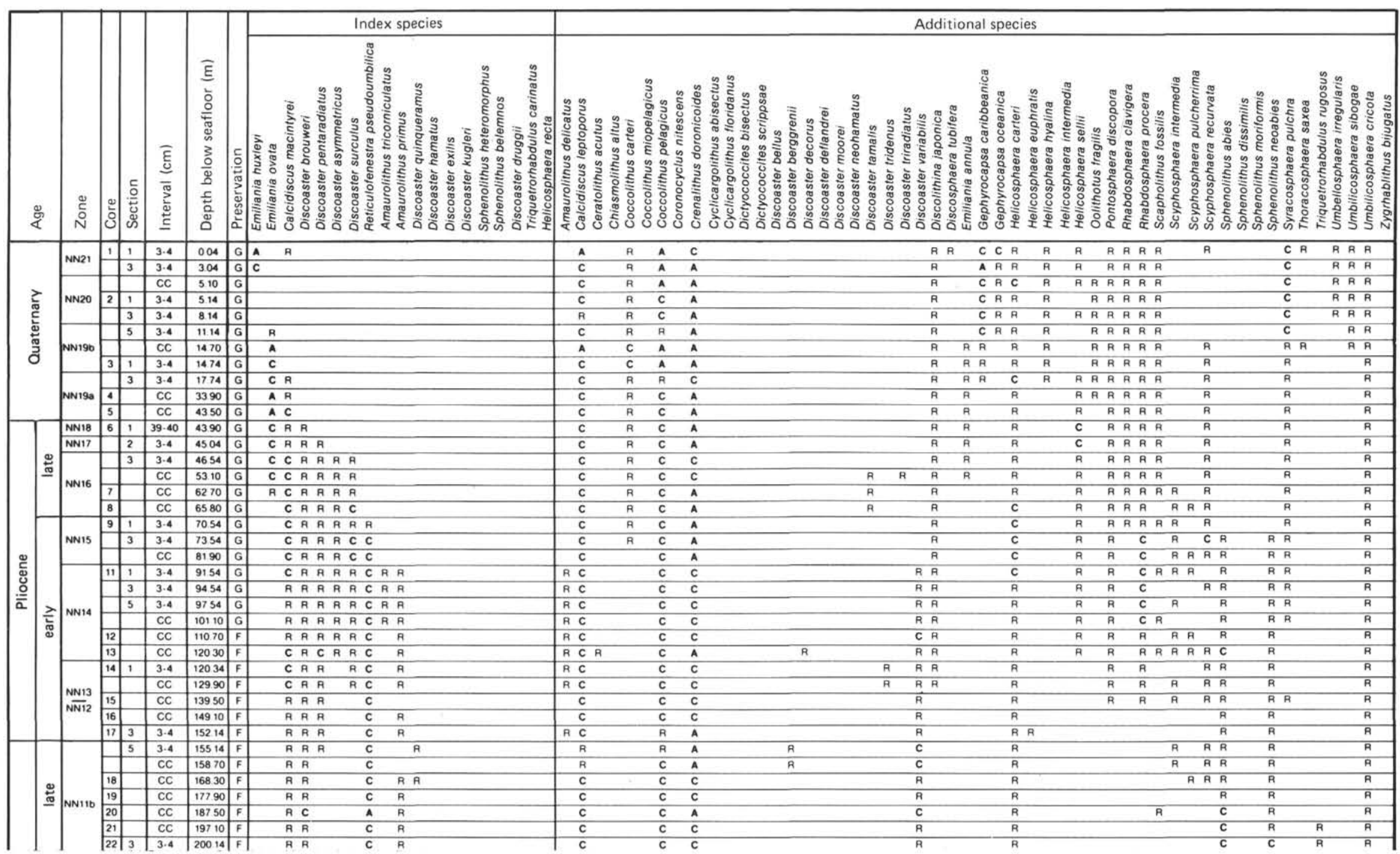




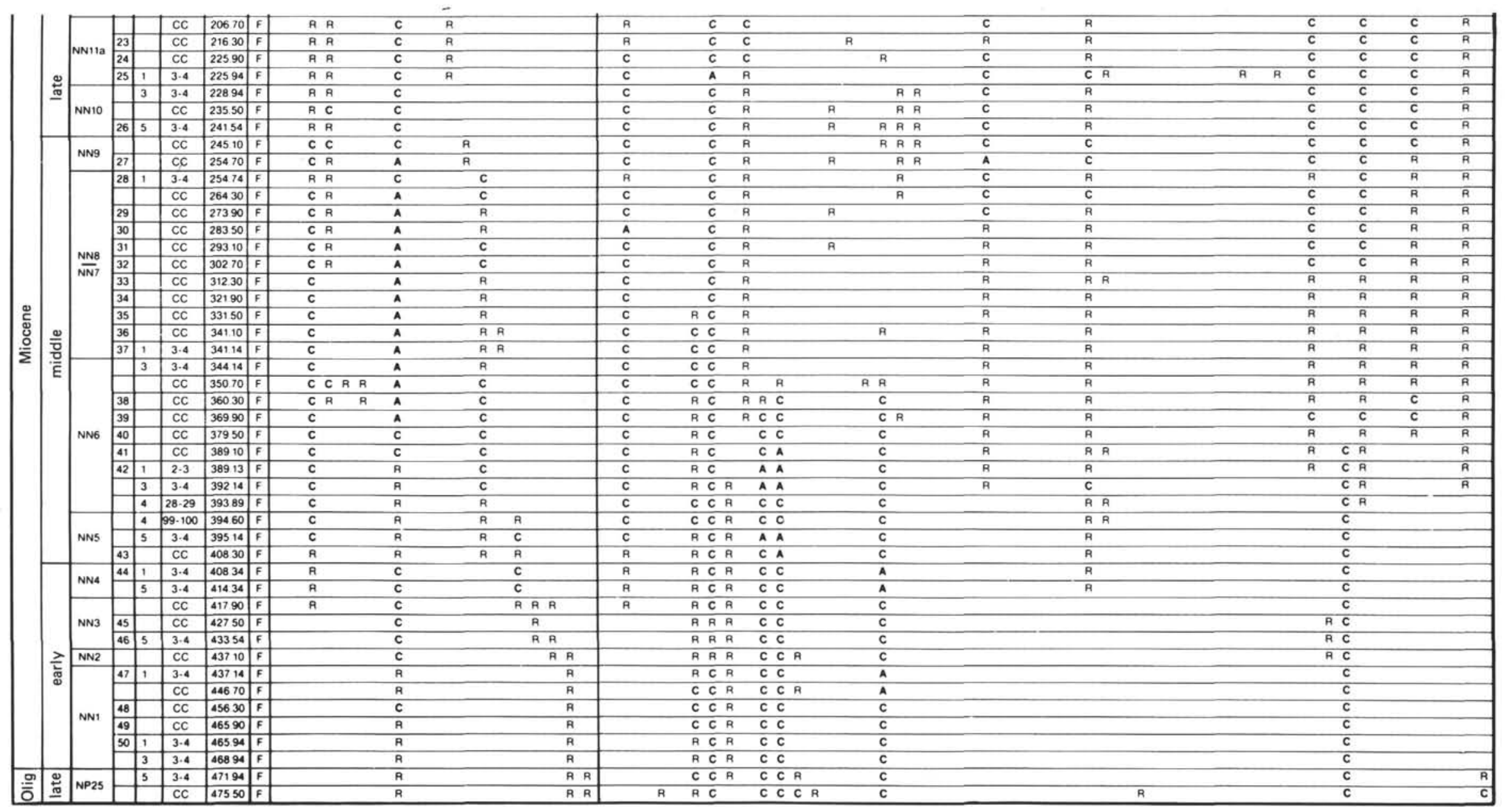




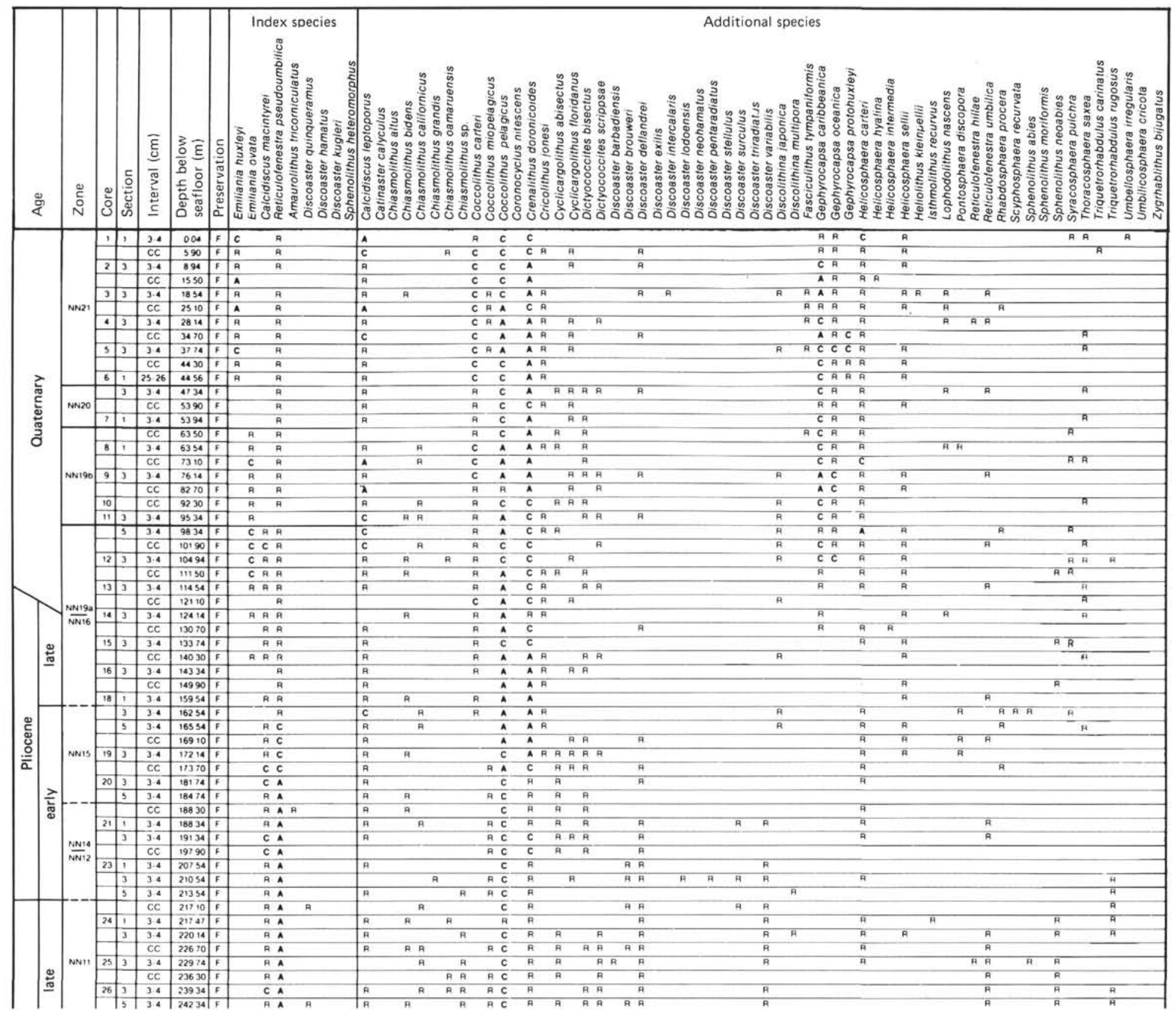




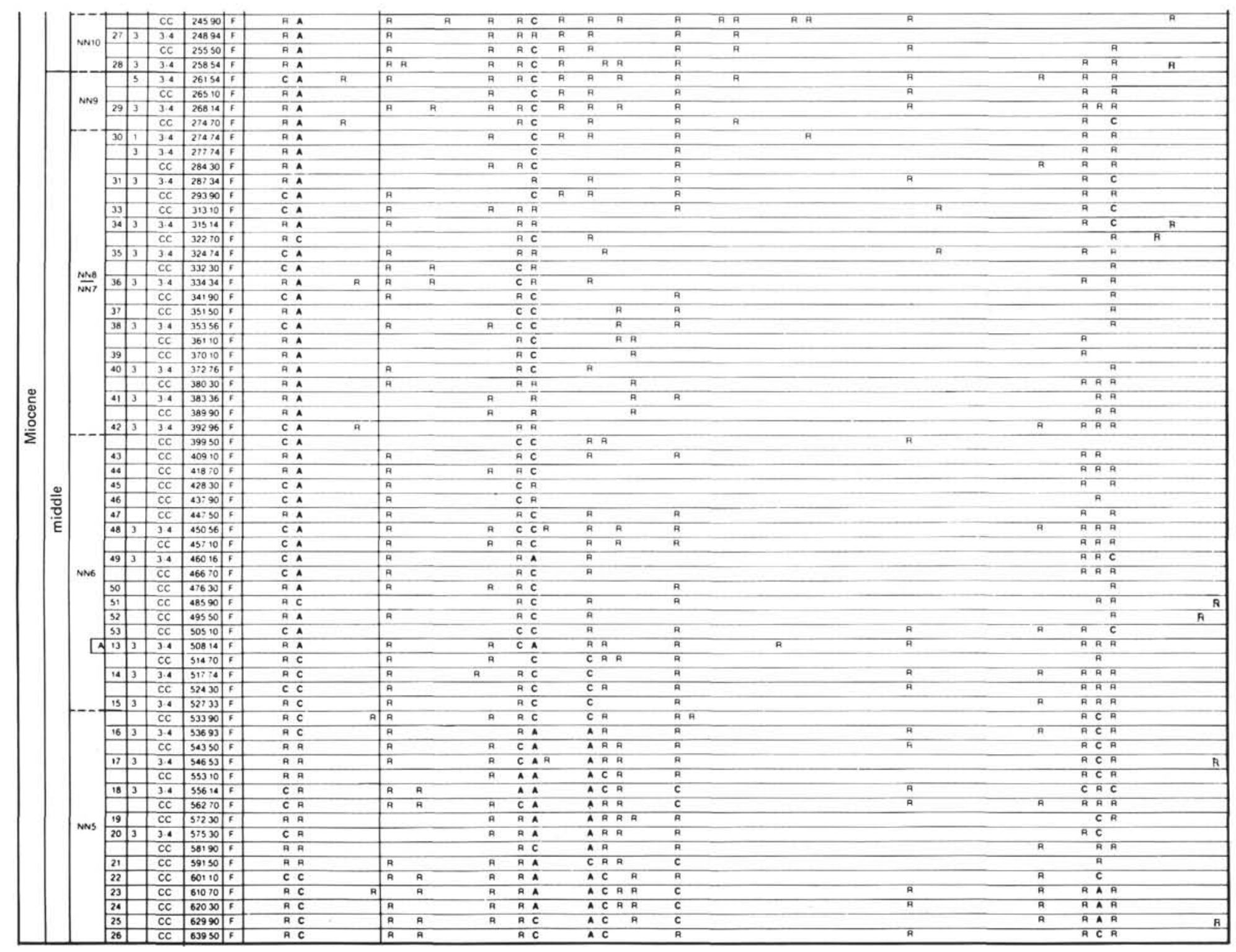


and Sphenolithus heteromorphus in Sample 594A-15,CC place Samples 594-42,CC through 594A-15-3, 3-4 cm in Zone NN6 and Samples 594A-15, CC through 594A$26, \mathrm{CC}$ in Zone NN5.

\section{ACKNOWLEDGMENTS}

I thank D. Bukry and S. Gartner for their review of the manuscript and for their helpful suggestions. Marathon Oil Company supported my participation on Leg 90 and approved publication of the results.

\section{REFERENCES}

Black, M., 1967. New names for some coccolith taxa. Proc. Geol. Soc. London, 1640:139-145.

Black, M., Barnes, B., 1959. The structure of coccoliths from the English Chalk. Geol. Mag., 96:321-328.

1961. Coccoliths and discoasters from the floor of the South Atlantic Ocean. J. R. Microsc. Soc., 80:137-147.

Boudreaux, J. E., and Hay, W. W., 1967. Zonation of the latest Pliocene-Recent interval. In Hay, W. W., Mohler, H. P., Roth, P. H., Schmidt, R. R., and Boudreaux, J. E., Calcareous Nannoplankton Zonation of the Cenozoic of the Gulf Coast and Caribbean-Antillean area, and Transoceanic Correlation. Trans. Gulf Coast Assoc. Geol. Soc., 17:428-480.

1969. Calcareous nannoplankton and biostratigraphy of the late Pliocene-Pleistocene-Recent sediments in the Submarex cores. Rev. Espan. Micropaleontol., 1:249-292.

Bramlette, M. N., and Riedel, W. R., 1954. Stratigraphic value of discoasters and some other microfossils related to Recent coccolithophores. J. Paleontol., 28:385-403.

Bramlette, M. N., and Sullivan, F. R., 1961. Coccolithophorids and related nannoplankton of the early Tertiary in California. Micropaleontology, 7:129-188.

Bramlette, M. N., and Wilcoxon, J. A., 1967. Middle Tertiary calcareous nannoplankton of the Cipero Section, Trinidad, W. I. Tulane Stud. Geol. Paleontol., 5:93-131.

Brönnimann, P., and Stradner, H., 1960. Die Foraminiferen und Discoasteriden-zonen von Kuba and ihre interkontinentale Korrelation. Erdoel-Z., 76:364-369.

Bukry, D., 1971a. Cenozoic calcareous nannofossils from the Pacific Ocean. San Diego Soc. Nat. Hist. Trans., 16:303-328. $17: 43-52$

1971b. Discoaster evolutionary trends. Micropaleontology,

1973a. Coccolith stratigraphy, eastern equatorial Pacific, Leg 16, Deep Sea Drilling Project. In van Andel, T. H., Heath, G. R., et al., Init. Repts. DSDP, 16:Washington (U.S. Govt. Printing Office), 653-711.

1973b. Low-latitude coccolith biostratigraphic zonation. In Edgar, N. T., Saunders, J. B., et al., Init. Repts. DSDP, 15: Washington (U.S. Govt. Printing Office), 685-703.

1973c. Phytoplankton stratigraphy, Deep Sea Drilling Project Leg 20, western Pacific Ocean. In Heezen, B. C., MacGregor, I. D., et al., Init. Repts. DSDP, 20: Washington (U.S. Govt. Printing Office), 307-317.

Bukry, D., and Bramlette, M. N., 1968. Stratigraphic significance of two genera of Tertiary calcareous nannofossils. Tulane Stud. Geol. Paleontol., 6:149-155.

1969. Some new and stratigraphically useful calcareous nannofossils of the Cenozoic. Tulane Stud. Geol. Paleontol., 7:131-142.

Bukry, D., and Percival, S. F., 1971. New Tertiary calcareous nannofossils. Tulane Stud. Geol. Paleontol., 8:123-146.

Cohen, C. L. D., 1964. Coccolithophorids from two Caribbean deepsea cores. Micropaleontology, 10:231-250.

1965. Coccoliths and discoasters from Adriatic bottom sediments. Leidse Geol. Meded., 35:1-44.

Cohen, C. L. D., and Reinhardt, P., 1968. Coccolithophorids from the Pleistocene Caribbean deep-sea core CP-28. N. Jb. Geol. Palaontol., Abhandl., 131:289-304.

Deflandre, G., 1942a. Coccolithophoridés fossiles d'Oranie. Genre Scyphosphaera Lohmann et Thorosphaera Ostenfeld. Soc. Hist. Nat. Toulouse, Bull., 77:125-137.

1942b. Possibilités morphogénétiques comparées du calcaire et de la silice, à propos d'un nouveau type de microfossile cal- caire de structure complexe, Lithostromation perdurum n.g. n. sp. C. R. Acad. Sci., 214:917-920.

1947. Braarudosphaera nov. gen., type d'une famille nouvelle de Coccolithophoridés actuels à éléments composites. $C$. $R$. Acad. Sci., 225:439-441.

1952. Classe des Coccolithophoridés (Coccolithophoridae Lohmann, 1902). In Grassé, P. P. (Ed.), Traité de Zoologie: Paris (Masson), 1:439-470.

1953. Hétérogénéité intrinsèque et pluralité des éléments dans les coccolithes actuels et fossiles. C. R. Acad. Sci., 237: $1785-1787$.

1959. Sur les nannofossiles calcaires et leur systématique. Rev. Micropaleontol., 2:127-152.

Deflandre, G., and Fert, C., 1954. Observations sur les Coccolithophoridés actuels et fossiles en microscopie ordinaire et électronique. Ann. Paleontol., 40:115-176.

Edwards, A. R, and Perch-Nielsen, K., 1975. Calcareous nannofossils from the southern southwest Pacific, Deep Sea Drilling Project, Leg 29. In Kennett, J. P., Houtz, R. E., et al., Init. Repts. DSDP, 29: Washington (U.S. Govt. Printing Office), 469-539.

Ellis, C. H., 1982. Calcareous nannoplankton biostratigraphy-Deep Sea Drilling Project Leg 60. In Hussong, D. M., Uyeda, S., et al., Init. Repts. DSDP, 60: Washington (U.S. Govt. Printing Office), 507-535.

Ellis, C. H., and Lohman, W. H., 1979. Neogene calcareous nannoplankton biostratigraphy in eastern Mediterranean deep-sea sediments (DSDP Leg 42A, Sites 375 and 376). Mar. Micropaleontol., 4:61-84.

Gaarder, K. R., 1970. Three new taxa of Coccolithineae. Nytt. Mag. Bot., 17:113-126.

Gartner, S., Jr., 1967. Calcareous nannofossils from Neogene of Trinidad, Jamaica, and Gulf of Mexico. Kans. Univ. Paleontol. Contr., Paper 29, 1-7.

1969a. Correlation of Neogene planktonic foraminifer and calcareous nannofossil zones. Trans. Gulf Coast Assoc. Geol. Soc., 19:585-599.

1969b. Phylogenetic lineages in the lower Tertiary coccolith genus Chiasmolithus. N. Am. Paleontol. Conv., Proc. G., 930-957. 1977. Calcareous nannofossil biostratigraphy and revised zonation of the Pleistocene. Mar. Microplaeontol., 2:1-25.

Gartner, S., Jr., and Bukry, D., 1974. Ceratolithus acutus Gartner and Bukry n.sp. and Ceratolithus amplificus Bukry and Percivalnomenclatural clarification. Tulane Stud. Geol. Paleontol., 11: 115-118.

1975. Morphology and phylogeny of the coccolithophycean family Ceratolithaceae. J. Res. U.S. Geol. Surv., 3:451-465.

Gran, H. H., and Braarud, T., 1935. A qualitative study of the phytoplankton in the Bay of Fundy and the Gulf of Maine. J. Biol. Board Canada, 1:279-467.

Haeckel, E., 1894. Systematische Phylogenie der Protisten und Pflanzen: Berlin (Reimer).

Haq, B. U., 1966. Electron microscope studies on some upper Eocene calcareous nannoplankton from Syria. Stockholm Contr. Geol., 15:23-37.

1973. Evolutionary trends in the Cenozoic coccolithophore genus Helicopontosphaera. Micropaleontology, 19:32-52.

Hay, W. W., and Mohler, H. P., 1967. Calcareous nannoplankton from early Tertiary rocks at Point Labau, France, and Paleoceneearly Eocene correlations. J. Paleontol., 41:1505-1541.

Hay, W. W., Mohler, H. P., Roth, P. H., Schmidt, R. R., and Boudreaux, J. E., 1967. Calcareous Nannoplankton Zonation of the Cenozoic of the Gulf Coast and Caribbean-Antillean area, and Transoceanic Correlation. Trans. Gulf Coast Assoc. Geol. Soc., 17.

Hay, W. W., Mohler, H. P., and Wade, M. E., 1966. Calcareous nannofossils from Nal'chik (northwest Caucasus). Eclogae Geol. Helv., 59:379-400.

Jafar, S. A., and Martini, E., 1975. On the validity of the calcareous nannoplankton genus Helicosphaera. Senckenberg. Lethaea, 56: 381-397.

Kamptner, E., 1927. Beitrag zur Kenntnis adriatischer Coccolithophoriden. Arch. Protistenk., 58:173-184.

1941. Die Coccolithineen der Südwestkuste von Istrien. Ann. Naturh. Mus. Wien, 51:54-149. 
1943. Zur revision der Coccolithineen-Spezies Pontosphaera huxleyi. Anz. Akad. Wiss. Wien., Math-Naturw., 80:43-49.

1948. Coccolithen aus dem Torton des Inneralpinen Wiener Beckens. Sitz-Ber. Osterr. Akad. Wiss., Math-Naturw. Kl., Abt. I, 157:1-16.

1950. Über den submikroskopischen Aufbau der Coccolithen. Anz. Osterr. Akad. Wiss., Math.-Naturw. Kl., 86:77-80.

1954. Untersuchungen über den Feinbau der Coccolithen.

Arch. Protistenk., 100:1-90.

1955. Fossile Coccolithineen-Skelettreste aus Insulinde. Eine mikropaläontologische Untersuchung. Verh. K. Nederl. Akad. Wet., Afd. Natuurk., ser. 2, 50:1-105.

1958. Betrachtungen zur Systematik der Kalkflagellaten, nebst Versuch einer neuen Gruppierung der Chrysomonadales. Arch. Protistenk., 103:54-116.

1963. Coccolithineen-Skelettreste aus Tiefseeablagerungen des Pazifischen Ozeans. Ann. Naturh. Mus. Wien, 66:139-204.

1967. Kalkflagellaten-Skelettreste aus Tiefseeschlamm des Sudatlantik Ozeans. Ann. Naturh. Mus. Wien, 71:117-198.

Levin, H. L., 1965. Coccolithophoridae and related microfossils from the Yazoo Formation (Eocene) of Mississippi. J. Paleontol., 39: 265-272.

Loeblich, A. R., and Tappan, H., 1963. Type fixation and validation of certain calcareous nannoplankton genera. Proc. Biol. Soc. Wash., 76:191-196.

1978. The coccolithophorid genus Calcidiscus Kamptner and its synonyms. J. Paleontol., 52:1390-1392.

Lohmann, H., 1902. Die Coccolithophoridae, eine monographie der coccolithen bildenden Flagellaten, zugleich ein Beitrag zür Kenntnis des Mittel-meerauftriebs. Arch. Protistenk., 1:89-165.

1912. Untersuchungen über das Pflanzen und Tierleben der Hoschsee. Zugleich ein Bericht über die biologischen Arbeiten auf der Fahrt der "Deutschland" von Bremerhaven nach Buenos Aires in der Zeit vom 7 Mai bis 7 September 1911. Univ. Berlin, Inst. Meeresk. Veroff., n. ser., Geogr. Naturw. Reihe, 1:1-92.

1919. Die Bevölkerung des Ozeans mit Plankton nach den Ergebnissen der Zentrifugenfänge während der Ausreise der "Deutschland" 1911. Arch. Biont. (Ges. Naturf. Freunde Berlin), 4.

McIntyre, A., 1970. Gephyrocapsa protohuxleyi sp. n., a possible phyletic link and index fossil for the Pleistocene. Deep-Sea Res., 17:187-190.

Markali, J., and Paasche, E., 1955. On two species of Umbellosphae$r a$, a new marine coccolithophorid genus. Nytt. Mag. Bot., 4: 95-100.

Martini, E., 1965. Mid-Tertiary calcareous nannoplankton from $\mathrm{Pa}-$ cific deep-sea cores. Symp. Colston Res. Soc. Proc. 17th. Symp., pp. 393-411.

1969. Nannoplankton aus dem Miozän von Gabon (Westafrika). N. J. Geol. Palaont., Abhandl., 132:285-300.

1971. Standard Tertiary and Quaternary calcareous nannoplankton zonation. In Farinacci, A. (Ed.), Proc II. Planktonic Conf., Roma, 1970, 2:739-785.

Martini, E., and Bramlette, M. N., 1963. Calcareous nannoplankton from the experimental Mohole drilling. J. Paleontol., 37:845-856.

Martini, E., and Ritzkowski, S., 1968. Was is das "Unter-Oligocän"? Nachr. Akad. Wiss. Gottingen, II Math.-Phys., Kl., Jahrg. 1968, pp. 231-249.
Martini, E., and Worsley, T., 1971. Tertiary calcareous nannoplankton from the western equatorial Pacific. In Winterer, E. L., Riedel, W. R., et al., Init. Repts. DSDP, 7, Pt. 2: Washington (U.S. Govt. Printing Office), 1471-1507.

Müller, C., 1970. Nannoplankton aus dem Mittel-Oligozän von Norddeutschland und Belgien. N. Jb. Geol. Palaontol., Abhandl., 135: 82-101.

1974. Calcareous nannoplankton, Leg 25 (western Indian Ocean). In Simpson, E. S. W., Schlich, R., et al., Init. Repts. $D S D P, 25$ : Washington (U.S. Govt. Printing Office), 579-633.

Murray, G., and Blackman, V. H., 1898. On the nature of the coccospheres and rhabdospheres. Phil. Trans. R. Soc. Lond., Ser. B, 190:427-441.

Norris, R. E., 1965. Living cells of Ceratolithus cristatus (Coccolithophorineae). Archiv. Protestenk., 108:19-24.

Okada, H., and Bukry, D., 1980. Supplementary modification and introduction of code numbers to the low-latitude coccolith biostratigraphic zonation (Bukry, 1973; 1975). Mar. Micropaleontol., 5: 321-325.

Okada, H., and McIntyre, A., 1977. Modern coccolithophores of the Pacific and North Atlantic Oceans. Micropaleontology, 23:1-55.

Ostenfeld, C. H., 1900. Über Coccosphaera. Zool. Anz., 23:198-200.

Perch-Nielsen, K., 1968. Der Feinbau und die Klassifikation der Coccolithen aus dem Maastrichten von Danemark. Biol. Skr. Dan. Vid. Selsk., 16:1-96.

Reinhardt, P., 1964. Einige Kalkflagellaten-Gattungen (Coccolithophoriden, Coccolithineen) aus dem Mesozoikum Deutschlands. Monatsber., Deutsch. Akad. Wiss. Berlin., 6:749-759.

Roth, P. H., 1973. Calcareous nannofossils-Leg 17, Deep Sea Drilling Project. In Winterer, E. L., Ewing, J. I., et al., Init. Repts. DSDP, 17: Washington (U.S. Govt. Printing Office), 675-795.

Schiller, J., 1925. Die planktonischen Vegetationen des adriatischen Meeres. A. Die Coccolithophoriden-Vegetation in den Jahren 19111914. Arch. Protistenk., 51:1-130.

1930. Coccolithineae. In Rabenhorst, L. (Ed.), Kryptogamen-Flora von Deutschland, Österreich und der Schweiz: Leipzig (Akad. Verlagsgesellschaft, 10:89-267.

Schwarz, E. H. L., 1894. Coccoliths. Ann. Mag. Nat. Hist., Ser. 6, 14:341-346.

Stradner, H., 1961. Vorkommen von Nannofossilien im Mesozoikum und Alttertiär. Erdoel-Z., 77:77-88.

Sullivan, F. R., 1964. Lower Tertiary nannoplankton from the California Coast Ranges. I. Paleocene. Calif. Univ. Publ. Geol. Sci., 44: 163-227.

Takayama, T., 1967. First report on nannoplankton of the Upper Tertiary and Quaternary of the Southern Kwanto Region, Japan. $J b$. Geol. Bundesanst. Wien, 110:169-198.

Tan, S. H., 1927. Over de samenstelling en het onstaan van krijt en mergelgesteenten van de Molukken. Jb. Mijnw. Nederl.-Indië, 55: 111-122.

Wallich, G. G., 1877. Observations on the coccosphaere. Ann. Mag. Nat. Hist., 19 (4th ser.):342-350.

Weber-van Bosse, A., 1901. Etudes sur les algues de l'Archipel Malaisien. III. Note préliminaire sur les résultats algologiques de l'expédition du Siboga. Ann. Jard. Bott. Bitenzorg, 17:126-141.

Date of Initial Receipt: 30 April 1984

Date of Acceptance: 22 August 1984 